\title{
In silico analysis of the endogenous time-keeping mechanism in citrus
}

\author{
Vera Quecini \\ Centro de Pesquisa e Desenvolvimento de Recursos Genéticos, Instituto Agronômico de Campinas, \\ Campinas, SP, Brazil.
}

\begin{abstract}
The endogenous time-keeping mechanism is responsible for organizing plant physiology and metabolism according to periodic environmental changes, such as diurnal cycles of light and dark and seasonal progression throughout the year. In plants, circadian rhythms control gene expression, stomatal opening, and the timing component of the photoperiodic responses, leading to enhanced fitness due to increased photosynthetic rates and biomass production. We have investigated the citrus genome databases of expressed sequence tags (EST) in order to identify genes coding for functionally characterized proteins involved in the endogenous time-keeping mechanism in Arabidopsis thaliana. Approximately 180,000 EST sequences from 53 libraries were investigated and 81 orthologs of clock components were identified. We found that the vast majority of Arabidopsis circadian clock genes are present in citrus species, although some important components are absent such as SRR1 and PRR5. Based on the identified transcripts, a model for the endogenous oscillatory mechanism of citrus is proposed. These results demonstrate the power of comparative genomics between model systems and economically important crop species to elucidate several aspects of plant physiology and metabolism.
\end{abstract}

Key words: central oscillator, circadian clock, data mining, photoperiodic responses, regulatory feedback loop.

Received: July 21, 2006; Accepted: April 17, 2007.

\section{Introduction}

The rotation of the earth on its axis causes alternating cycles of light and dark at a period of approximately $24 \mathrm{~h}$. The physiology, metabolism and behavior of most organisms are drastically affected by these daily environmental changes that lead to apparent biological oscillations known as diurnal rhythms. The conceptual model of circadian clocks consists of an entrainment or input pathway that synchronizes the pacemaker with its environmental surroundings, a central oscillator that generates and sustains rhythmicity, and multiple output pathways that link the oscillator to the organism's physiology and metabolism (Bell-Pedersen et al., 2005).

In plants, the entraining stimulus is mainly light, with a smaller contribution from temperature (Salomé and McClung, 2005; Edwards et al., 2006). Accordingly, the input pathway to the plant clock consists mostly of photoreceptor families (Millar, 2004), ubiquitously found in plants, including economically important species (Santelli and Sivieiro, 2001; Hecht et al., 2005).

The central oscillatory mechanism of all known circadian clocks is dependent on autoregulatory feedback loops

Send correspondence to Vera Quecini. Centro de Pesquisa e Desenvolvimento de Recursos Genéticos, Instituto Agronômico de Campinas, Caixa Postal 28, 13001-970 Campinas, SP, Brazil. E-mail: vquecini@iac.sp.gov.br.
(Bell-Pedersen et al., 2005). In plants, the Arabidopsis circadian clock mechanism has been partially elucidated. However, circadian control of metabolism and physiology is evident in most plant species, including citrus, where several economically important features are circadianly regulated, such as flavonoid and terpenoid metabolism regulation and essential oil composition (Lee and Castle, 2001; Lin et al., 2002; Frizzo et al., 2004). Moreover, gene expression and developmental processes in citrus have also been demonstrated to be under control of an endogenous time-keeping mechanism (Smith and Wareing, 1972; Abied and Holland, 1994).

The negative limb of the Arabidopsis clock consists of two highly similar single MYB-domain factors CCA1

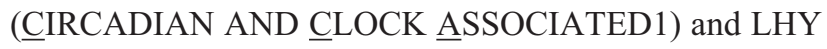

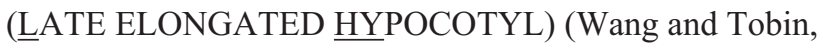
1998). The positive limb is the pseudo-response regulator TOC1 (TIMING $\underline{\text { OF }} \underline{\mathrm{CAB}} 1$ ), also called PRR1 (Arabidopsis thaliana PSEUDO-ㅌESPONSE $\underline{\text { REGULA- }}$ TOR 1), its orthologs PRR5, PRR7 and PRR9 (Makino et al., 2000; Strayer et al., 2000; Alabadí et al., 2001; Mizoguchi et al., 2002), the MYB transcription factor LUX ARRHYTHMO (LUX)/PHYTOCLOCK 1 (PCL1) (Hazen et al., 2005, Onai and Ishiura, 2005) and the novel protein ELF4 (EARLY FLOWERING 4) (Kikis et al., 2005). 
The oscillatory mechanism depends on CCA1- and LHY-binding to the evening element (EE, AAATATCT) in the TOC1 promoter, which represses transcription of the response regulator-like gene (Alabadí et al., 2001). During the day, the levels of CCA1 and LHY decrease, releasing their repression on TOCl that peaks at dusk when the levels of CCA1 and LHY are at their trough. Subsequently, the increasing levels of TOC1 during the evening induce its function as a positive regulator of $C C A 1$ and $L H Y$ expression through an as yet uncharacterized mechanism (Alabadí et al., 2001, Matsushika et al., 2002; Mizoguchi et al., 2002; Harmer and Kay, 2005; Hazen et al., 2005). Several other gene products are required for $C C A 1$ and $L H Y$ expression, including GIGANTEA (GI), ELF3, ELF4, and LUX (Park et al., 1999; Doyle et al., 2002; Mizoguchi et al., 2002; Hazen et al., 2005); thus, generating the interlocked feedback loops. The second proposed loop consists of TOC1 transcriptional activation by GI, which in turn, is repressed by TOC1 (Locke et al., 2005). A third autoregulatory loop is hypothesized to consist of CCA1 and LHY as positive regulators of TOC1-family members $P R R 5, P R R 7$, and PRR9 (Farré et al., 2005; Harmer and Kay, 2005). Currently, distinct lines of evidence support the idea that PRR5/7/9 are negative regulators of CCA1/LHY: (i) CCA1 and $L H Y$ transcripts accumulate in $\operatorname{prr} 7$ and $\operatorname{prr} 7$ prr 9 mutants (Farré et al., 2005) and, (ii) CCA1 is constitutively transcribed in the arrhythmic prr 5 prr 7 prr 9 triple mutant (Nakamichi et al., 2005b). Moreover, PRR5/7/9 and TOC1 are thought to be mutually repressive and partially redundant (Farré et al., 2005; Nakamichi et al., 2005a; Salomé and McClung, 2005).

Post-transcriptional regulation is involved in several processes of circadian timing in plants. CCA1 and LHY are phosphorylated by casein kinase II (CK2) in a manner similar to the pace-control mechanism of the mammalian circadian clock (Sugano et al., 1998, Sugano et al., 1999). The robustness of LHY oscillations has been attributed to posttranslational modifications and proteasome-mediated degradation (Kim et al., 2003; Song and Carré, 2005). A second type of posttranslational modification, implied in clock function, is protein acetylation; mutants lacking the $\mathrm{N}$-acetylglucosamine transferase activity of SPINDLY (SPY) exhibit altered leaf movement rhythms (Tseng et al., 2004). Recent evidence has demonstrated the crucial role of light- and clock-controlled proteolysis for the plant endogenous clock. The novel family of putative photoreceptors ZEITLUPE (ZTL), LOV KELCH PROTEIN 2 (LKP2) and FLAVIN-BINDING KELCH REPEAT F-BOX 1 (FKF1) provides a direct link between the central oscillator and ubiquitin-mediated protein degradation (Nelson et al., 2000; Somers et al., 2000; Schultz et al., 2001). ZTL is a component of the Skp1-Cullin-F-box (SCF) complex that recruits TOC1 for proteasomal degradation (Somers et al., 2000; Más et al., 2003b; Han et al., 2004).
Day length is measured by the integration of circadian clock and light perception information at the level of CONSTANS (CO) protein expression and activity (Suárez-López, 2001; Yanovsky and Kay, 2002; Imaizumi et al., 2003; Valverde et al., 2004; Imaizumi et al., 2005). CO directly regulates the expression of FLOWERING LOCUS $\underline{T}(F T)$ in Arabidopsis and of its homolog $\underline{H E A D I N G} \underline{D A T E}$ $3 a(H D 3 a)$ in rice (Putterill et al., 1995; Kobayashi et al., 1999; Samach et al., 2000; Onouchi et al., 2000; Izawa et al., 2002; Kojima et al., 2002), which promotes the expression of meristem-identity genes triggering the transition from vegetative to reproductive development at the shoot apical meristem. The expression and activity of $\mathrm{CO}$ is regulated by the internal circadian clock of the plant, so that $C O$ mRNA transcription is restricted to $12 \mathrm{~h}$ after dawn until around dawn of the following day (Suárez-Lopez et al., 2001; Valverde et al., 2004). Under long day conditions, $C O$ mRNA is therefore present during the day, and when the $\mathrm{CO}$ protein is translated it is stabilized in the nucleus of the cell and will activate the expression of other genes (An et al., 2004; Valverde et al., 2004). Under short day conditions, $C O$ mRNA is only transcribed during the night because the days are shorter than 12-h long, and the dark translated $\mathrm{CO}$ protein is rapidly broken down, and unable to activate gene expression (Valverde et al., 2004). Thus, early flowering of Arabidopsis occurs in the long days of spring and early summer through the coincidence of circadian clock control of $C O$ mRNA expression and direct exposure of the plant to light (Yanovsky and Kay, 2002; Valverde et al., 2004). In rice, $C O$ gene homolog, called $\underline{H E A D I N G} \underline{D} A T E 1$ (HDI) also has a key role in flower timing control. However, the function of $H D 1$ in rice is reversed, thus, promoting flowering on short days. The rice $\mathrm{CO}$ homolog protein is activated at the end of a long day; but instead of activating other genes as it does in Arabidopsis, HD1 represses gene activity and flowering under longer photoperiods (Yano et al., 2001; Izawa et al., 2002). In contrast, under short day conditions HD1 repression is absent, allowing HD3a to promote flowering (Onouchi et al., 2000; Izawa et al., 2002; Kojima et al., 2002). In temperate forest trees, the CO/FT regulatory module has been demonstrated to control of flowering time in response to variations in daylength; and, unexpectedly, it also controls the short-day-induced growth cessation and bud set occurring in the fall (Böhlenius et al., 2006). Thus, annual species and trees appear to have a common time keeping mechanism to control development during the progression of the seasons. The conserved functionality of key meristem identity genes between Arabidopsis and citrus has been demonstrated (Endo et al., 2005; Pillitteri et al., 2004; Pena et al., 2001). However, the regulatory mechanisms of their induction, including light quality and photoperception, temperature and day length changes remain elusive. 
Recent evidence has demonstrated that photoperiodic induction of synchronous vegetative bud break or flowering is also common in tropical plants (Borchert et al., 2005). At tropical latitudes, changes in day length that are large enough to affect plant development occur around the equinoxes. Therefore, a single annual period of synchronous flowering after the autumn equinox indicates the induction of flowering in 'short-day plants' in response to declining day length; whereas, 'long-day plants' flowering and vegetative bud break is induced by an increasing day length that occurs after the spring equinox (Borchert et al., 2005). The molecular mechanism leading to day length measuring in the tropics has been hypothesized to involve the integration of photoperception and the endogenous clock as observed in temperate species (Borchert et al., 2005).

The present work was designed to investigate the circadian temporal programming and its integrative pathways to physiology and metabolism in citrus, employing combined in silico EST profiling and domain structural data analysis. The results presented here demonstrate that the vast majority of the gene products involved in circadian timing in Arabidopsis are present in citrus species. Moreover, genetic distance and domain structure analyses have uncovered extensive amino acid sequence conservation, providing indications of functional equivalence of Arabidopsis and citrus transcripts. Taken together, the results have demonstrated the feasibility of incorporating in silico analyses for gene discovery in non-model species.

\section{Material and Methods}

\section{Database searches and alignments}

Homologs of the Arabidopsis thaliana circadian clock were identified in BLAST searches (Altschul et al., 1997) against citrus EST databases (CitEST), which consist of approximately 180,000 ESTs obtained from the sequencing of 53 specific libraries. Data validation was performed by tBLASTx and tBLASTn searches of the retrieved sequence against the locally built GenBank database at CitEST. Sequences failing to retrieve the original sequence used to query the database were eliminated from the projects. The resulting alignments were filtered by a threshold e-value of 1e-15 and the hits were further analyzed according to functional domain description. Validated sequences were translated and protein (deduced amino acid) alignments were performed using ClustalX (Thompson et al., 1997). When necessary, alignments were manually adjusted using Lasergene MegAlign (DNASTAR, Madison, WI, USA).

\section{Motif analysis and in silico characterization}

The identified homologs were further investigated for the presence and sequence conservation of recognizable functional domains described in several protein analysis and gene function databases (European Bioinformatics Institute-European Molecular Biology Laboratory - EMBLEBI; Expert Protein Analysis System - ExPaSy from Swiss Institute of Bioinformatics - SIB; Gene Ontology database GO; Protein Families database - Pfam).

\section{Phylogenetic analysis}

The functionality of citrus genes in comparison to their Arabidopsis counterparts was assessed by genetic distance and phylogenetic studies. Phylogenetic analyses were performed using distance and parsimony methods in the software PAUP* 4.0b10, using the software default parameters. Resampling bootstrap trees containing 1000 random samples were constructed using PSIGNFIT software. Modular functional domains were employed for genetic distance studies for genes previously characterized as having divergent regions and conserved blocks.

\section{In silico gene expression analysis}

Qualitative gene expression profiling was performed by in silico analyses of the afore mentioned citrus EST databases using virtual northern blot analyses. The gene of interest was used in queries against reference sequence databases, generating an alignment of the input gene to its paralogs. The resulting alignment was then used to find sequences in the entire mRNA input that are specific to the gene (probe). The resulting alignments were collectively used to query the EST database again using BLAST. This heuristic attempts to avoid false-positives, or ESTs from a paralog of the input gene rather than the gene itself. The identity numbers of the ESTs matching the probes were recovered and CitEST was used to find the names of the libraries from which those ESTs were derived. The frequency of reads of each EST contig in a given library was calculated and normalized according to the total number of reads from the investigated library and the total number of reads in all libraries. A correlation matrix between EST contigs and libraries was then generated and gene expression patterns among ESTs and libraries were obtained by hierarchical clustering based on a Spearman Rank correlation matrix using Cluster v.2.11 software (Eisen et al., 1998), by substituting the clusters by their average expression pattern. Graphic outputs were generated using Tree View v.1.6 software and presented in grayscale.

\section{Results and Discussion}

The essential components of the circadian clock input pathway, gating mechanism, central oscillator, the output and photoperiod integrative pathways from Arabidopsis thaliana, and to a lesser extent Oryza sativa, were compiled and the protein sequences of the main components were employed to search citrus EST databases. The identified hits were ranked according to the deduced amino acid sequence identity to the functionally characterized protein used as bait and further analyzed for their functionality. 
This way, 81 citrus ESTs and EST contigs that are similar to components of the endogenous time-keeping mechanism and its ancillary regulation loops were identified and functionally assigned to gene ontology classes (Figure 1). From this total, 20 transcripts correspond to input pathway photoreceptors, proteins involved in the gating mechanism and in ancillary entrainment loops in plants (Table 1). Twenty-nine citrus sequences showed extensive sequence conservation to components of the central oscillator mechanism (Table 2) and 32 resemble proteins involved in the integration between the pacemaker and plant metabolism and physiology (Table 3). Interestingly, some important components of the time keeping-mechanism from Arabidopsis are absent from citrus sequence databases, such as the input gene SRR1 (AT5G59560; Staiger et al., 2003) and the TOC1/PRR1-quintet member PRR5 (AT5G24470, Nakamichi et al., 2005a). Moreover, citrus and Arabidopsis gene families, including the DOF transcription factor CDF1 and MADS-box CONSTANS, appear to have undergone differential expansion events.

\section{Input pathway}

\section{Light entrainment pathway}

Four of the five Arabidopsis phytochromes (PHYA, PHYB, PHYD and PHYE) function in an additive manner in the red-light (R) input to the clock (Devlin and Kay, 2000). CRYPTOCHROME 1 (CRY1) acts as the main clock input photoreceptor under high and low fluences of blue light (B) whereas both CRY1 and CRY2 redundantly function at intermediate fluences of B (Devlin and Kay, 2000; Somers et al., 1998). Surprisingly, clock entrainment under white light seems to be dependent on the physical interaction of CRY2 and PHYB and their localization in nuclear speckles (Más et al., 2000). Moreover, cryl mutant plants also show altered entrainment under red light suggesting that CRY1 is required for PHYA signaling to the clock in both R and B (Devlin and Kay, 2000). Quadruple Arabidopsis mutants lacking phyA, phyB, cryl and cry 2 are able to maintain circadian rhythmicity (Yanovsky et al., 2000), suggesting that additional components participate in the light input pathway to reset the clock. The rhythmic expression of PHY and CRY genes (Tóth et al., 2001) has been hypothesized to contribute to the gating mechanism on the input to the clock, although the bulk PHY protein levels do not oscillate (Sharrock and Clack, 2002).

Loss-of-function $z t l$ mutants show fluence-rate dependent period alteration, indicating that ZTL has a role in the light input pathway (Somers et al., 2000). ZTL mRNA abundance is not clock regulated, but its protein levels peak around dusk and reach trough levels around dawn (Kim et al., 2003). The rate of proteasome-mediated degradation of ZTL varies during the course of the day: ZTL is more stable at dusk, close to its peak value, and is more rapidly degraded at dawn when it reaches its trough. F-box proteins provide specificity to proteasomal degradation pathways
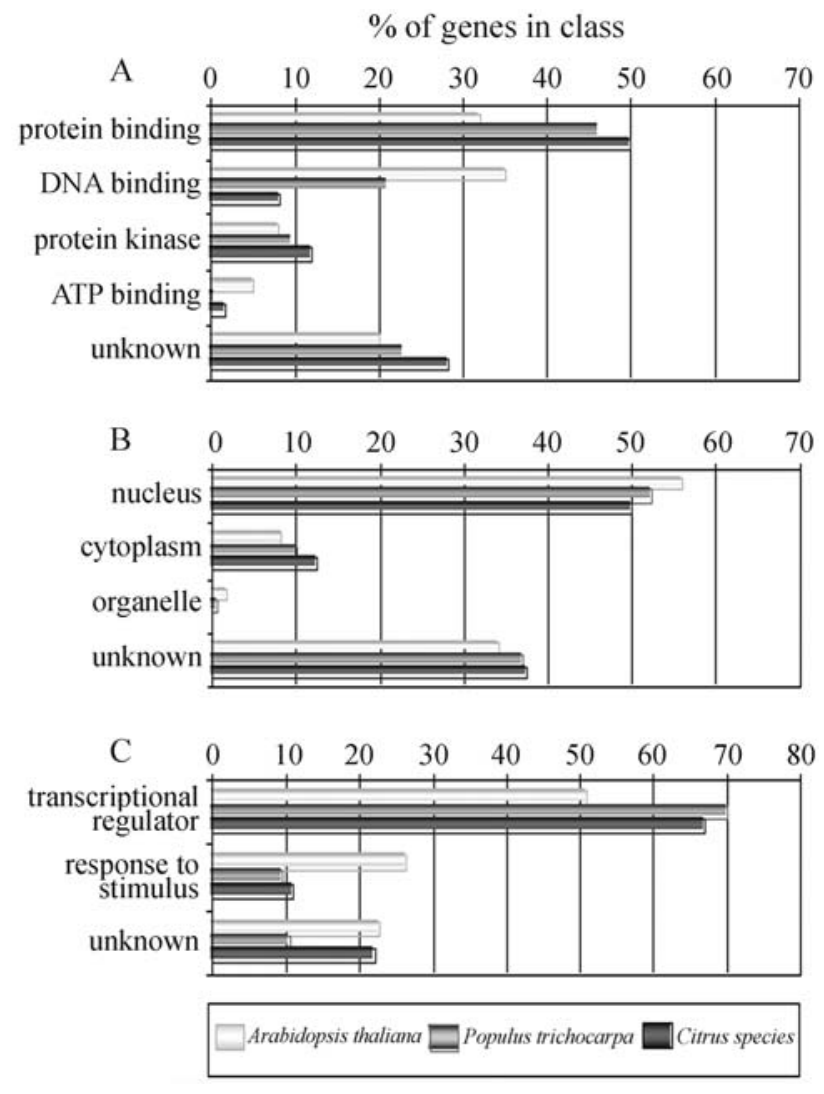

Figure 1 - Functional classification of citrus transcripts associated to the endogenous time-keeping mechanism using gene ontology. A. molecular function; B. cellular component; C. biological process. Assignments are based on the data available at the TIGR Arabidopsis thaliana Gene Index version 13.0.

by interaction and polyubiquitination-mediated degradation of determined targets. ZTL has been demonstrated to recruit the oscillator component TOC1 for degradation (Somers et al., 2000; Más et al., 2003b; Han et al., 2004). In ztl mutants, TOC1 levels are elevated and only weakly rhythmic, demonstrating that ZTL is critical for TOC1 proteolysis and rhythmicity. Correspondingly, ZTL overexpression induces a dosage-dependent period shortening (Han et al., 2004). Citrus photoreceptor families and the components of their proteolytic degradation pathway have been extensively discussed in the accompanying paper on photoperception and light signal transduction.

\section{Gating mechanism}

In Arabidopsis, light-conditional arrhythmic behavior is observed in the absence of ELF3 (EARLY FLOWERING 3) and TIC (TIME FOR COFFEE) proteins (Hicks et al., 1996; Hicks et al., 2001; McWatters et al., 2000; Covington et al., 2001; Liu et al., 2001; Hall et al., 2003). Interestingly, elf3 null mutants are arrhythmic at different phases of the oscillation when compared to tic mutants and double elf 3 tic mutants lack rhythmicity under light and dark conditions (Hall et al., 2003). Thus, these 
Table 1 - Citrus ESTs with homology to genes involved in the input pathway to the circadian clock in Arabidopsis thaliana.

\begin{tabular}{|c|c|c|c|c|c|c|}
\hline \multicolumn{2}{|c|}{ Arabidopsis thaliana } & \multicolumn{3}{|c|}{ CitEST } & \multirow{2}{*}{$\begin{array}{l}\text { Protein motifs } \\
\text { and biological process }\end{array}$} & \multirow[t]{2}{*}{ References } \\
\hline $\mathrm{Name}^{\mathrm{a}}$ & Gene & EST (n. reads) ${ }^{\mathrm{b}}$ & $\%^{\mathrm{c}}$ & e value & & \\
\hline \multicolumn{7}{|c|}{ Entrainment photoreceptors } \\
\hline CRYI & AT4G08920 & $\begin{array}{l}\text { C10-CR (2) } \\
\text { S1-CS }\end{array}$ & $\begin{array}{c}23.3(\mathrm{P}: 48 \%) \\
74.6(\mathrm{FL})\end{array}$ & $\begin{array}{l}3 e-91 \\
1 e-106\end{array}$ & $\begin{array}{l}\text { FAD binding domain, DNA photo- } \\
\text { lyase, B photoreceptor, circadian } \\
\text { clock entrainment }\end{array}$ & Cashmore et al., 1999 \\
\hline PHYA & AT1G09570 & $\begin{array}{l}\text { S2-CS } \\
\text { S3-CS }\end{array}$ & $\begin{array}{l}58.3(\mathrm{FL}) \\
34.7(\mathrm{FL})\end{array}$ & $\begin{array}{l}6 e-86 \\
4 e-81\end{array}$ & $\begin{array}{l}\text { PAS1, PAS2, chromophore binding } \\
\text { domain, HKL domain, R/FR photo- } \\
\text { receptor }\end{array}$ & $\begin{array}{c}\text { Sharrock and } \\
\text { Quail, } 1989\end{array}$ \\
\hline PHYB & AT2G18790 & $\begin{array}{l}\text { S4-CR } \\
\text { S5-CS }\end{array}$ & $\begin{array}{c}15.3(\mathrm{FL}) \\
8.1(\mathrm{P}: 72 \%)\end{array}$ & $\begin{array}{l}3 e-75 \\
3 e-55\end{array}$ & $P H Y$ family & Boylan et al., 1994 \\
\hline$F K F 1$ & AT1G68050 & $\begin{array}{c}\mathrm{C} 5-\mathrm{CS}(2) \\
\mathrm{C} 6-\mathrm{CR} / \mathrm{CS}(3)\end{array}$ & $\begin{array}{l}76.3(\mathrm{FL}) \\
19.3(\mathrm{FL})\end{array}$ & $\begin{array}{c}1 \mathrm{e}-142 \\
1 \mathrm{e}-46\end{array}$ & $\begin{array}{l}\text { Kelch repeats, F-box domain, LOV } \\
\text { domain, putative } \\
\text { photoreceptor, photoperiodic flow- } \\
\text { ering control, circadian clock }\end{array}$ & $\begin{array}{l}\text { Nelson et al., 2000; } \\
\text { Imaizumi et al., } 2005\end{array}$ \\
\hline$L K P 2$ & AT2G18915 & S6-CS & $22.0(\mathrm{FL})$ & $1 e-76$ & $Z T L / F K F 1 / L K P 2$ family & Schultz et al., 2001 \\
\hline$Z T L$ & AT5G57360 & $\mathrm{C} 5-\mathrm{CS}(2)$ & $29.7(\mathrm{FL})$ & $8 \mathrm{e}-71$ & $Z T L / F K F 1 / L K P 2$ family & Somers et al., 2000 \\
\hline \multicolumn{7}{|c|}{ Ancillary factors } \\
\hline ELF3 & AT2G25930 & $\begin{array}{l}\mathrm{C} 1-\mathrm{CS} / \mathrm{CR}(2) \\
\mathrm{C} 2-\mathrm{CR}(2) \\
\text { S7-CA } \\
\text { S8-PT }\end{array}$ & $\begin{array}{c}9.8(\mathrm{FL}) \\
10.6(\mathrm{FL}) \\
19.4(\mathrm{FL}) \\
7.5(\mathrm{FL})\end{array}$ & $\begin{array}{l}6 e-24 \\
1 \mathrm{e}-18 \\
2 \mathrm{e}-20 \\
1 \mathrm{e}-15\end{array}$ & $\begin{array}{l}\text { novel nuclear protein, hydro- } \\
\text { xyproline-rich glycoprotein family } \\
\text { protein, zeitnehmer, } \\
\text { rhythmic input to the clock }\end{array}$ & Carré, 2002 \\
\hline$G I$ & AT1G22770 & $\begin{array}{c}\mathrm{C} 1-\mathrm{CS}(6) \\
\mathrm{C} 2-\mathrm{CS} / \mathrm{CR}(2) \\
\mathrm{C} 3-\mathrm{CG} / \mathrm{CR} / \mathrm{CS} \\
(4) \\
\text { S9-CS } \\
\text { S10-CR }\end{array}$ & $\begin{array}{c}31.2(\mathrm{FL}) \\
25.4(\mathrm{P}: 73 \%) \\
25.4(\mathrm{FL}) \\
15.3(\mathrm{P}: 76 \%) \\
18.8(\mathrm{P}: 73 \%)\end{array}$ & $\begin{array}{c}5 \mathrm{e}-144 \\
2 \mathrm{e}-45 \\
1 \mathrm{e} 131 \\
2 \mathrm{e}-56 \\
5 \mathrm{e}-55\end{array}$ & $\begin{array}{l}\text { putative membrane spanning do- } \\
\text { mains, nuclear localization, phyB } \\
\text { signal transduction, photoperiodic } \\
\text { control of flowering time }\end{array}$ & Fowler et al., 1999 \\
\hline PIF3 & AT1G09530 & C6-CG/LT (2) & $25.5(\mathrm{FL})$ & $4 e-27$ & $\begin{array}{l}\text { transcriptional regulator, bHLH do- } \\
\text { main, photomorphogenesis }\end{array}$ & Ni et al., 1999 \\
\hline
\end{tabular}

${ }^{\mathrm{a}}$ Gene name abbreviations: CRY: cryptochrome; DASH: Drosophila, Arabidopsis, Synechocistis, human; ELF: early flowering; FKF1: F-box, Kelch repeat, Flavin-binding protein1; GI: GIGANTEA; LKP2: LOV domain, Kelch repeat protein2; PCL: phytoclock; PHY: phytochrome; PIF: phytochrome interacting factor; ZTL: Zeitlupe.

${ }^{\mathrm{b}} \mathrm{C}$ : contig (number); S: singlet (number); CA: Citrus aurantium; CG: Citrus aurantifolia; CR: Citrus reticulata; CS: Citrus sinensis; LT: Citrus latifolia; PT: Poncirus trifoliata.

${ }^{c}$ Identity percentage at the amino acid level; (FL), full-length cDNA; (P), partial cDNA: percentage of Arabidopsis cDNA.

${ }^{\mathrm{d}}$ Functional domain abbreviations: FAD: flavina adenosine dinucleotide; LOV: light, oxygen, voltage subtype PAS domain; PAS: Per, ARNT, Sym domain; HKL: histidine kinase-like.

proteins are likely to be responsible for the gating mechanism at different times of the day and are termed Zeitnehmer (from German, time taker). The ELF3 protein contains no recognizable domains and the TIC gene remains to be identified (Liu et al., 2001; Hall et al., 2003). In citrus transcriptome, two EST contigs from Citrus sinensis and $C$. reticulata, one from C. aurantium and one from Poncirus trifoliata, sharing moderate sequence similarity to ELF3, were identified (Table 2). The lack of functionally characterized domains in Arabidopsis ELF3 prevents further considerations about the role of the citrus homologs.

\section{Temperature entrainment pathway}

Temperature signaling to the clock is much less well defined; however, gene expression and cotyledon movement have been demonstrated to be effectively entrained by temperature cycles in Arabidopsis (Michael and McClung,
2002; Salomé et al., 2002; Salomé and McClung, 2005). The pseudo-response regulators PRR7 and PRR9 are essential for temperature-mediated clock synchronization, as prr 7 prr 9 double mutants fail to entrain to temperature cycles that are effective to entrain wild type plants (Salomé and McClung, 2005). Citrus genome has three cDNAs similar to PRR7, two from C. sinensis and one from P. trifoli$a t a$, and two EST contigs highly similar to PRR9 (Table 3). In Arabidopsis and rice, these proteins participate in the central oscillator mechanism as well (Farré et al., 2005; Murakami et al., 2005; Nakamichi et al., 2005b). The citrus PRR homologs are discussed in-depth in the following section. Interestingly, the photoperiodic transcriptional activator, GIGANTEA (GI) and the MADS-box protein FLC have been assigned to a quantitative trait locus (QTL) responsible for a substantial portion of natural variation in temperature-mediated circadian clock entrainment (Ed- 
Table 2 - Citrus ESTs with homology to genes involved in the central oscillator of the circadian clock in Arabidopsis thaliana.

\begin{tabular}{|c|c|c|c|c|c|c|}
\hline \multicolumn{2}{|c|}{ Arabidopsis thaliana } & \multicolumn{3}{|c|}{ CitEST } & \multirow{2}{*}{$\begin{array}{l}\text { Protein motifs } \\
\text { and biological process }\end{array}$} & \multirow[t]{2}{*}{ References } \\
\hline $\mathrm{Name}^{\mathrm{a}}$ & Gene & EST (n. reads) ${ }^{\mathrm{b}}$ & $\%^{\mathrm{c}}$ & e value & & \\
\hline$C C A 1$ & AT2G46830 & $\begin{array}{c}\text { C3-CS (2) } \\
\text { C7-CS/PT (8) } \\
\text { C16-PT (4) } \\
\text { C18-CS/CR (4) } \\
\text { S11-CR }\end{array}$ & $\begin{array}{l}23.3(\mathrm{FL}) \\
24.6(\mathrm{FL}) \\
65.2(\mathrm{FL}) \\
23.4(\mathrm{FL}) \\
64.5(\mathrm{FL})\end{array}$ & $\begin{array}{l}9 \mathrm{e}-46 \\
4 \mathrm{e}-35 \\
2 \mathrm{e}-51 \\
2 \mathrm{e}-26 \\
2 \mathrm{e}-36\end{array}$ & $\begin{array}{l}\text { MYB-related transcription factor, } \\
\text { circadian rhythms, central oscillator } \\
\text { component, negative feedback loop }\end{array}$ & $\begin{array}{l}\text { Green and Tobin, 1999; } \\
\text { Wang and Tobin, } 1998\end{array}$ \\
\hline$C K 2$ & AT3G60250 & $\begin{array}{l}\text { C2-CR/CS (6) } \\
\text { S12-CG } \\
\text { S13-CR } \\
\text { S14-CS }\end{array}$ & $\begin{array}{l}26.2(\mathrm{FL}) \\
64.9(\mathrm{FL}) \\
16.9(\mathrm{FL}) \\
16.4(\mathrm{FL})\end{array}$ & $\begin{array}{l}1 e-34 \\
6 e-43 \\
3 e-30 \\
1 e-32\end{array}$ & $\begin{array}{l}\text { regulatory }(\beta) \text { subunit of the protein } \\
\text { kinase } C K 2 \text {, circadian rhythms reg- } \\
\text { ulator, transcription factor binding } \\
\text { and phosphorylation }\end{array}$ & $\begin{array}{l}\text { Sugano et al., 1998; } \\
\text { Sugano et al., } 1999\end{array}$ \\
\hline $\begin{array}{l}\text { DET1/ } \\
\text { FUS2 }\end{array}$ & AT4G10180 & $\begin{array}{l}\text { S15-CS } \\
\text { S16-LT }\end{array}$ & $\begin{array}{c}14.3(\mathrm{FL}) \\
12.5(\mathrm{P}: 87 \%)\end{array}$ & $\begin{array}{l}3 e-55 \\
2 e-57\end{array}$ & $\begin{array}{c}\text { nuclear localized } \\
\text { photomorphogenesis repressor }\end{array}$ & Chory and Peto, 1990 \\
\hline ELF4 & AT2G40080 & $\begin{array}{l}\text { S17-CR } \\
\text { S18-CS } \\
\text { S19-PT }\end{array}$ & $\begin{array}{l}40.9(\mathrm{FL}) \\
11.4(\mathrm{FL}) \\
11.4(\mathrm{FL})\end{array}$ & $\begin{array}{l}2 e-32 \\
3 e-28 \\
1 e-27\end{array}$ & $\begin{array}{l}\text { no recognizable domain, circadian } \\
\text { clock central oscillator ancillary } \\
\text { loop, photomorphogenesis }\end{array}$ & $\begin{array}{l}\text { Doyle et al., 2004; } \\
\text { Kikis et al., } 2005\end{array}$ \\
\hline$L H Y$ & AT1G01060 & $\begin{array}{c}\mathrm{C} 11-\mathrm{CS} / \mathrm{CR} \\
(22) \\
\text { C14-PT (2) } \\
\text { S20-PT } \\
\text { S21-CR }\end{array}$ & $\begin{array}{l}26.5(\mathrm{FL}) \\
16.9(\mathrm{FL}) \\
20.1(\mathrm{FL}) \\
16.8(\mathrm{FL})\end{array}$ & $\begin{array}{l}1 \mathrm{e}-55 \\
6 \mathrm{e}-40 \\
3 \mathrm{e}-36 \\
5 \mathrm{e}-32\end{array}$ & $\begin{array}{l}\text { MYB-related transcription factor, } \\
\text { circadian rhythms, central oscillator } \\
\text { component, negative feedback loop }\end{array}$ & $\begin{array}{c}\text { Schaffer } \text { et al., 1998; } \\
\text { Green and Tobin, } 1999\end{array}$ \\
\hline $\begin{array}{l}L U X \\
\text { /PCL1 } \\
\text { family }^{\mathrm{e}}\end{array}$ & AT3G46640 & $\begin{array}{c}\text { C1-CS (6) } \\
\text { (LUX3) } \\
\text { C2- CS/PT (3) } \\
\text { (LUX/PCL) } \\
\text { S22-CR } \\
\text { (LUX5) } \\
\text { S23-CR } \\
\text { (LUX5) }\end{array}$ & $\begin{array}{c}12.9(\mathrm{FL}) \\
11.5(\mathrm{FL}) \\
14.2(\mathrm{P}: 45 \%) \\
30.7(\mathrm{P}: 75 \%)\end{array}$ & $\begin{array}{l}4 e-72 \\
6 e-54 \\
8 e-52 \\
1 e-54\end{array}$ & $\begin{array}{c}\text { single MYB DNA-binding domain } \\
\text { (type SHAQKYF), putative activa- } \\
\text { tor of CCA } 1 / \text { LHY expression, re- } \\
\text { pressed by CCA } 1 / \text { LHY }\end{array}$ & $\begin{array}{l}\text { Hazen } \text { et al., 2005; } \\
\text { Onai and Ishiura, 2005 }\end{array}$ \\
\hline $\begin{array}{l}\text { PRR1/ } \\
\text { TOC1 }\end{array}$ & AT5G61380 & S24-CS & 68.0 (P: 85\%) & $5 e-66$ & $\begin{array}{l}\text { pseudo-response regulator, response } \\
\text { regulator receiver domain, circadian } \\
\text { rhythm, photomorphogenesis }\end{array}$ & Alabadi et al. 2001 \\
\hline PRR3 & AT5G60100 & $\begin{array}{c}\mathrm{C} 3-\mathrm{CS} / \mathrm{CR} / \mathrm{LT} \\
(8) \\
\mathrm{S} 25-\mathrm{CS} \\
\mathrm{S} 26-\mathrm{CS}\end{array}$ & $\begin{array}{l}72.0(\mathrm{FL}) \\
70.7(\mathrm{FL}) \\
64.5(\mathrm{FL})\end{array}$ & $\begin{array}{l}2 \mathrm{e}-37 \\
1 \mathrm{e}-21 \\
2 \mathrm{e}-22\end{array}$ & $P R R$ family & $\begin{array}{l}\text { Matsushita et al., 2002; } \\
\text { Murakami et al., } 2004\end{array}$ \\
\hline PRR7 & AT5G02810 & $\begin{array}{l}\text { S27-CS } \\
\text { S28-CS } \\
\text { S29-PT }\end{array}$ & $\begin{array}{c}68.1(\mathrm{P}: 73 \%) \\
59.2(\mathrm{FL}) \\
56.5(\mathrm{FL})\end{array}$ & $\begin{array}{l}3 e-31 \\
9 e-22 \\
6 e-25\end{array}$ & $P R R$ family & Nakamichi et al., 2005 \\
\hline PRR9 & AT2G46790 & $\begin{array}{c}\mathrm{C} 1-\mathrm{CS}(2) \\
\mathrm{C} 2-\mathrm{CS} / \mathrm{CR}(3)\end{array}$ & $\begin{array}{l}80.0(\mathrm{FL}) \\
77.6(\mathrm{FL})\end{array}$ & $\begin{array}{l}2 \mathrm{e}-39 \\
6 \mathrm{e}-29\end{array}$ & $P R R$ family & Nakamichi et al., 2005 \\
\hline$S P Y$ & AT3G11540 & $\begin{array}{c}\text { C1-CS/CR (7) } \\
\text { S30-CR } \\
\text { S31-CS }\end{array}$ & $\begin{array}{l}27.4 \text { (P: } 91 \%) \\
25.0 \text { (P: 75\%) } \\
15.5 \text { (P: 77\%) }\end{array}$ & $\begin{array}{l}2 e-65 \\
4 e-43 \\
3 e-28\end{array}$ & $\mathrm{~N}$-acetyl glucosamine transferase & Tseng et al., 2004 \\
\hline
\end{tabular}

${ }^{a}$ Gene name abbreviations: PRR: Arabidopsis pseudo response regulator; $C C A$ : circadian and clock associated; $C K$ : casein kinase II regulatory subunit; $E L F$ : early flowering; $L H Y$ : long hypocotyl; SPY: spindly; TOC: timing of CAB.

${ }^{\mathrm{b}} \mathrm{C}$ : contig (number); S: singlet (number); CA: Citrus aurantium; CG: Citrus aurantifolia; CR: Citrus reticulata; CS: Citrus sinensis; LT: Citrus latifolia; PT: Poncirus trifoliata.

${ }^{c}$ Identity percentage at the amino acid level.

${ }^{\mathrm{d}}$ Functional domain abbreviations: FAD: flavina adenosine dinucleotide; LOV: light, oxygen, voltage subtype PAS domain; PAS: Per, ARNT, Sym domain; HKL: histidine kinase-like.

${ }^{e}$ LUX/PCL1 family members in Arabidopsis thaliana: AT5G59570, AT5G05090, AT3G10760, AT2G40970.

wards et al., 2005). Five citrus transcripts, whose deduced amino acid sequences share significant homology to GI and GI-like proteins, and three FLC homologs were identified (Table 2 and Table 3, respectively). Thus, the Arabidopsis components of the temperature-mediated clock entrainment pathway are present in citrus transcriptome databases.

\section{Central oscillator}

The simplified model for the transcriptional/translational negative-feedback loop of the Arabidopsis circadian clock consists of the reciprocal regulation between the highly similar MYB-type transcription factors CCA1/LHY in the negative limb and TOC1 and the novel protein ELF4 
A

CCA1/LHY

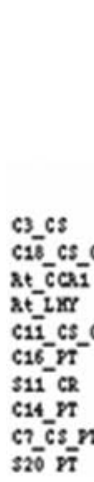

$-1 x$

C11_Cs_CR

$16 \mathrm{nt}$

14 YT

$320 \mathrm{nt}$

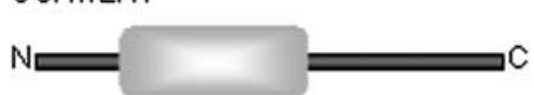

MYB (SHAQK motif)

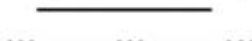

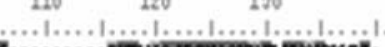

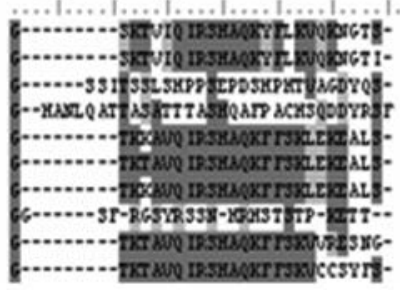

46r5:-

B

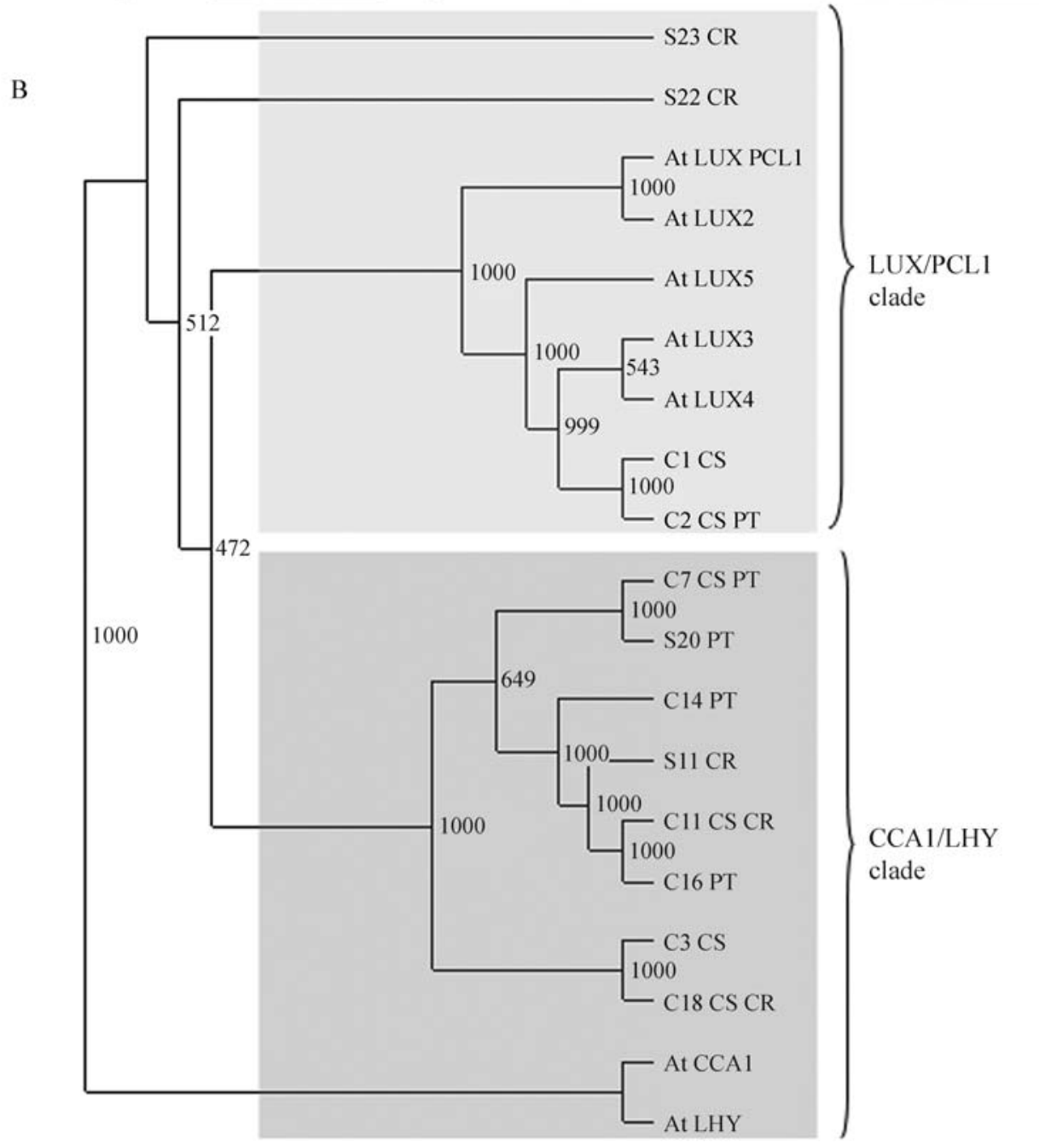

\section{LUXJPCL1}

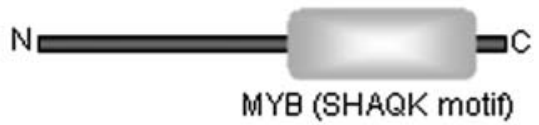

At_LtX_pet:

at_tox2

cics

C2 $63 n$

at $t \overline{V_{3}}$

st_tox4

at_tuxs

$52 \overline{2}$ CR

$\$ 23 \mathrm{CR}$

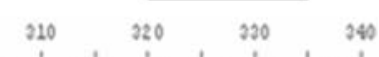

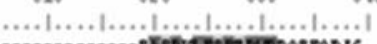

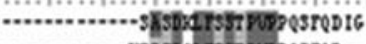

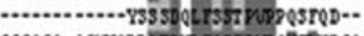

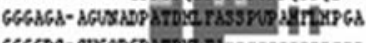

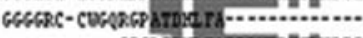
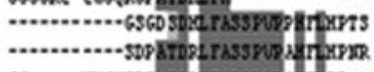

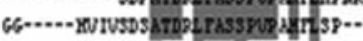

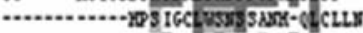

............

Figure 2 - MYB-type transcription factors involved in the circadian clock in citrus and $A$. thaliana. A. Schematic representation of CCA1/LHY and LUX protein domains and MYB domain alignment in citrus and A. thaliana. B. Phylogenic analysis of citrus and Arabidopsis CCA1/LHY and LUX protein families. Neighbor-joining tree for citrus deduced amino acid and Arabidopsis full length sequences were aligned with ClustalX. Bootstrap values are indicated above each branch. At, Arabidopsis thaliana; C Number, contig number; CS, Citrus sinensis; CR, Citrus reticulata; PT, Poncirus trifoliata.

in the positive limb (Strayer et al., 2000; Kikis et al., 2005; McClung, 2006). In citrus transcriptome databases, nine homologs to the TOC1/PRR1 family of pseudo-response regulators, nine cDNAs whose deduced amino acid sequences are similar to the MYB-type transcription factors CCA1 and LHY, and three similar to ELF4 were present
(Table 3). Moreover, in silico analyses have identified four homologs of the LUX/PCL1 family, which are hypothesized to function on an interlocked loop to activate CCA1 and LHY transcription (Hazen et al., 2005; Onai and Ishiura, 2005), four homologs of CK2, a casein kinase responsible for CCA1 phosphorylation (Daniel et al., 2004) 
Table 3 - Citrus ESTs with homology to genes involved in the integration of the circadian clock mechanism and the generation of output responses in Arabidopsis thaliana.

\begin{tabular}{|c|c|c|c|c|c|c|}
\hline \multicolumn{2}{|c|}{ Arabidopsis thaliana } & \multicolumn{3}{|c|}{ CitEST } & \multirow{2}{*}{$\begin{array}{l}\text { Protein motifs }{ }^{\mathrm{d}} \\
\text { and biological process }\end{array}$} & \multirow[t]{2}{*}{ References } \\
\hline $\mathrm{Name}^{\mathrm{a}}$ & Gene & EST (n. reads) ${ }^{\mathrm{b}}$ & $\%^{\mathrm{c}}$ & e value & & \\
\hline $\begin{array}{l}C D F 1 \text { and } \\
C D F \text { family }\end{array}$ & AT5G62430 & $\begin{array}{c}\text { C4-CS/PT (3) } \\
\text { C10- CA/CR (2) } \\
\text { S1-CS } \\
\text { S2-PT } \\
\text { S3-PT } \\
\text { S4-PT }\end{array}$ & $\begin{array}{l}33.3(\mathrm{FL}) \\
36.9(\mathrm{FL}) \\
43.2(\mathrm{FL}) \\
33.3(\mathrm{FL}) \\
16.7(\mathrm{FL}) \\
16.7(\mathrm{FL})\end{array}$ & $\begin{array}{l}3 \mathrm{e}-37 \\
4 \mathrm{e}-46 \\
3 \mathrm{e}-56 \\
2 \mathrm{e}-45 \\
6 \mathrm{e}-31 \\
5 \mathrm{e}-46\end{array}$ & $\begin{array}{l}\text { DOF-type zinc finger, H-protein promo- } \\
\text { ter binding factor-2a, interacts with LKP2 } \\
\text { and FKF1, repressor of CONSTANS }\end{array}$ & Imaizumi et al., 2005 \\
\hline $\mathrm{CO}$ & AT5G15840 & $\mathrm{C} 5-\mathrm{CG} / \mathrm{CS}(4)$ & $54.7(\mathrm{FL})$ & $3 e-58$ & $\begin{array}{l}\text { Zn finger C2HC5 B-box DNA binding } \\
\text { motif, CCT motif }\end{array}$ & $\begin{array}{l}\text { Putterill et al., 1995, } \\
\text { Suárez-López et al., } \\
2001\end{array}$ \\
\hline $\begin{array}{l}\text { COL1 and } \\
\text { COL family }\end{array}$ & AT5G15850 & $\begin{array}{c}\text { C4-CS/CR (24) } \\
\text { (COL2) } \\
\text { C1-CS (2) } \\
\text { (COL7) } \\
\text { C7-CR (2) } \\
\text { (COL16) } \\
\text { C13-PT(2) } \\
\text { (COL5) } \\
\text { C14-CR/CA (3) } \\
\text { (COL15) } \\
\text { C15-LT (2) } \\
\text { (COL7) } \\
\text { S1-CS (COL1) } \\
\text { S2-CS (COL7) } \\
\text { S3-PT (COL1) } \\
\text { S4-CR (COL13) } \\
\text { S5-CL (COL4) } \\
\text { S6-CS (COL4) } \\
\text { S7-CS (COL2) } \\
\text { S8-PT (COL7) }\end{array}$ & $\begin{array}{c}62.9(\mathrm{FL}) \\
40.0(\mathrm{FL}) \\
38.4(\mathrm{FL}) \\
29.3(\mathrm{FL}) \\
35.1(\mathrm{FL}) \\
29.4(\mathrm{FL}) \\
57.0(\mathrm{FL}) \\
50.7(\mathrm{FL}) \\
48.4(\mathrm{P}: 87 \%) \\
42.6(\mathrm{FL}) \\
37.5(\mathrm{FL}) \\
31.6(\mathrm{P}: 77 \%) \\
29.3(\mathrm{FL}) \\
27.8(\mathrm{FL})\end{array}$ & $\begin{array}{l}7 \mathrm{e}-83 \\
1 \mathrm{e}-39 \\
8 \mathrm{e}-39 \\
1 \mathrm{e}-25 \\
9 \mathrm{e}-28 \\
2 \mathrm{e}-26 \\
5 \mathrm{e}-79 \\
3 \mathrm{e}-70 \\
2 \mathrm{e}-67 \\
6 \mathrm{e}-64 \\
2 \mathrm{e}-31 \\
4 \mathrm{e}-30 \\
1 \mathrm{e}-25 \\
7 \mathrm{e}-24\end{array}$ & CONSTANS family & $\begin{array}{l}\text { Robson et al., 2001, } \\
\text { Griffiths et al., } 2003\end{array}$ \\
\hline ELF7 & AT1G79730 & $\mathrm{C} 1-\mathrm{CR} / \mathrm{LT}(3)$ & $19.7(\mathrm{FL})$ & $1 e-26$ & $\begin{array}{l}\text { PAF1 homolog (yeast complex associated } \\
\text { to RNA pol II, FLC and FLM regulator }\end{array}$ & He et al., 2004 \\
\hline$E L F 8$ & AT2G06210 & C1-CS (4) & $34.4(\mathrm{FL})$ & $1 e-43$ & $\begin{array}{l}\text { CTR9 homolog (yeast PAF1 com- } \\
\text { plex), FLC expression induction }\end{array}$ & He et al., 2004 \\
\hline$F D$ & AT4G35900 & S1-CG & $17.8(\mathrm{FL})$ & $5 e-16$ & $\begin{array}{l}\text { bZIP transcription factor, flowering pro- } \\
\text { moter, interacts with FT }\end{array}$ & $\begin{array}{l}\text { Abe et al., 2005; Wigge } \\
\text { et al., } 2005\end{array}$ \\
\hline$F L C$ & AT5G10140 & $\begin{array}{l}\text { C1-CS (4) } \\
\text { S1-CS } \\
\text { S2-CS }\end{array}$ & $\begin{array}{c}40.8(\mathrm{FL}) \\
37.8(\mathrm{FL}) \\
39.3(\mathrm{P}: 83 \%)\end{array}$ & $\begin{array}{l}7 \mathrm{e}-33 \\
7 \mathrm{e}-25 \\
8 \mathrm{e}-23\end{array}$ & $\begin{array}{l}\text { MADS-box protein, K-box transcription } \\
\text { factor, floral transition repressor, temper- } \\
\text { ature integration to circadian clock }\end{array}$ & Edwards et al., 2006 \\
\hline $\begin{array}{l}\text { FT/ } \\
\text { TSF }\end{array}$ & $\begin{array}{l}\text { AT1G65480 } \\
\text { AT4G20370 }\end{array}$ & S1-CS & $\begin{array}{l}23.5(\mathrm{FL}) \\
21.7(\mathrm{FL})\end{array}$ & $\begin{array}{l}2 \mathrm{e}-51 \\
2 \mathrm{e}-51\end{array}$ & $\begin{array}{l}\text { phosphatidylethanolamine-binding pro- } \\
\text { tein motif, induced by long days in } \\
\text { Arabidopsis, flowering promoter, target } \\
\text { of CO and acts upstream of SOC1 }\end{array}$ & $\begin{array}{l}\text { Izawa et al., 2002, } \\
\text { Halliday et al., } 2003\end{array}$ \\
\hline PFT1 & AT1G25540 & $\mathrm{C} 2-\mathrm{CS} / \mathrm{CR}(6)$ & $68.6(\mathrm{P}: 53 \%)$ & $1 \mathrm{e}-148$ & $\begin{array}{l}\text { von Willebrand factor type } \\
\text { A, glutamine-rich C-terminal }\end{array}$ & Cerdán and Chory, 2003 \\
\hline SOC1 & AT2G45660 & $\begin{array}{l}\text { S1-PT } \\
\text { S2-PT }\end{array}$ & $\begin{array}{l}22.4 \text { (P: 75\%) } \\
22.5 \text { (P: 77\%) }\end{array}$ & $\begin{array}{l}1 \mathrm{e}-51 \\
1 \mathrm{e}-51\end{array}$ & $\begin{array}{l}\text { K-box region, SRF-type transcription } \\
\text { factor, bZIP DNA binding motif, plant } \\
\text { DUF } 1319\end{array}$ & Onouchi et al., 2000 \\
\hline
\end{tabular}

${ }^{\mathrm{a}}$ Gene name abbreviations: $C D F$ : cycling DOF factor; $C O$ : CONSTANS; $C O L$ : CONSTANS-like, $E L F$ : early flowering; $F D$ : flowering locus D; $F L C$ : flowering locus C; FT: flowering locus T; PFT: phytochrome and flowering time; SOC: suppressor of CONSTANS; TSF: twin sister of FT.

${ }^{\mathrm{b}} \mathrm{C}$ : contig (number); S: singlet (number); CA: Citrus aurantium; CG: Citrus aurantifolia; CR: Citrus reticulata; CS: Citrus sinensis; LT: Citrus latifolia; PT: Poncirus trifoliata.

${ }^{\mathrm{c} I d e n t i t y ~ p e r c e n t a g e ~ a t ~ t h e ~ a m i n o ~ a c i d ~ l e v e l ; ~(F L), ~ f u l l-l e n g t h ~ c D N A ; ~(P), ~ p a r t i a l ~ c D N A: ~ p e r c e n t a g e ~ o f ~ A r a b i d o p s i s ~ c D N A . ~}$

${ }^{\mathrm{d}}$ Functional domains abbreviations: bZIP: basic leucine zipper; CCT: CONSTANS, CONSTANS-like and TOC1; CTR: calcitonin receptor, DOF: DNA binding with one finger, DUF: domain of function unknown; MADS:, MCM1, AGAMOUS, DEFICIENS and SRF; PAF: platelet-activating factor; SRF: serum response factor; Zn finger: zinc finger DNA binding domain.

${ }^{\mathrm{e}} \mathrm{CDF} 1$ family members in Arabidopsis thaliana: AT3G47500, AT5G39660.

${ }^{\mathrm{f} C O L}$ family members in Arabidopsis thaliana: AT5G15850, AT3G02380, AT1G04500, AT3G07650 , AT1G07050 , AT1G49130 , AT2G24790, AT2G33350, AT3G12890, AT3G21150, AT3G21890, AT4G15248, AT5G24930, AT5G48250, AT5G53420, AT1G06040. 
and two SPY-like sequences (Table 3). Thus, at least two interlocked feedback regulatory transcription/ translation loops of the Arabidopsis central oscillator and two post-translational modification systems are present and likely to be functional in a citrus endogenous time-keeping mechanism.

Citrus transcriptome analyses revealed the presence of 13 cDNAs corresponding to Arabidopsis MYB-type of transcriptional regulators involved in the circadian clock central oscillator mechanism; namely, CCA1, LHY and the LUX/PCL1 family (Table 3, Figure 2). CCA1/LHY-like sequences are highly similar in $C$. sinensis, $C$. reticulata and $P$. trifoliata, sharing up to $62.2 \%$ of sequence identity (Figure 2A). Moreover, the sequences are highly similar to their Arabidopsis counterparts (Table 2, Figure 2A). Interestingly, the single-MYB LUX/PCL1 family in citrus is more divergent and appears to have undergone a differential expansion process (Figure 2B), as the highest sequence identity to Arabidopsis LUX proteins was of $30.7 \%$ and only four members were found, in contrast to the five members identified in Arabidopsis (Hazen et al., 2005). In silico transcriptional analysis of citrus MYB-like genes with similarities to clock components demonstrated a high frequency of CCA1/LHY and LUX homologs in developing fruits and a similar expression pattern (Figure 2). However, the normalized levels of $L U X$ transcripts were approximately 1.5 times smaller than the levels observed for CCA1/LHY (Figure 3).

The Arabidopsis PRR family consists of TOC1/PRR1 and four other members (PRR3, PRR5, PRR7 and PRR9) whose structural designs are very similar (Matsushika et al., 2000) and include a receiver-like (or pseudo-receiver) domain at their $\mathrm{N}$-terminus followed by a long specific intervening sequence, followed by another motif of about 50 amino acids at the very C-terminal end that is shared by the PRR family, termed the CCT mo-

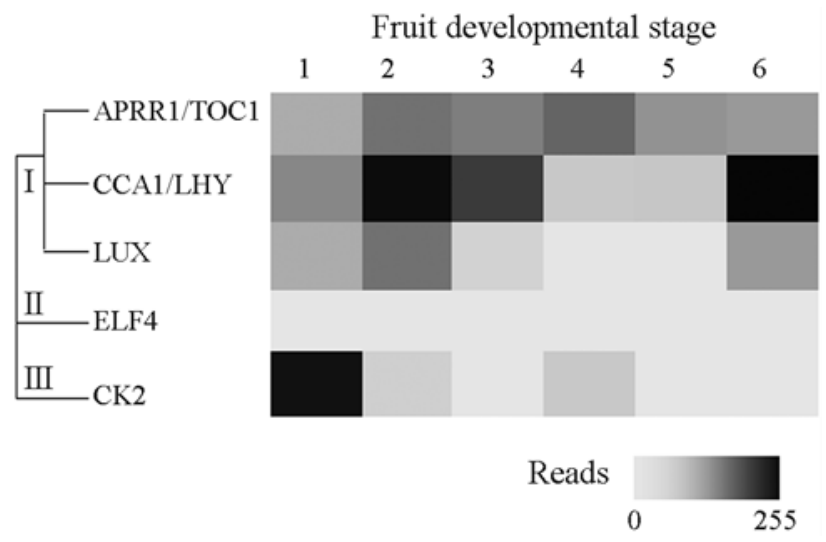

Figure 3 - In silico expression profile of the components of the central oscillator mechanism of the endogenous clock in citrus. The normalized number of reads for the transcripts in each library is represented as a grayscale. Fruit developmental stage libraries are represented as columns. Hierarchical clustering of the expression patterns is represented by roman numerals.

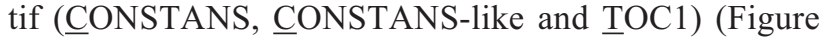
4A) (Mizuno and Nakamichi, 2005). In citrus, eight transcripts sharing extensive sequence similarity to Arabidopsis and Oryza sativa PRR were identified (Table $3)$. The identified transcript sequences are highly conserved at the CCT region and more divergent at the response regulator domain (Figure 4B, Figure 4C). Citrus PRR family is highly represented in fruit developmental stage libraries and shows an expression profile similar to CCA1/LHY and LUX (Figure 3). Interestingly, the transcripts of $P R R s$ begin to accumulate after subjective dawn in the following order PRR9-PRR7-PRR5-PRR3-PRR1, separated by 2-3 h intervals (Matsushika et al., 2000). In Oryza sativa, $P R R$ family also consists of 5 members that present sequential transcriptional induction from subjective dawn and with approximately $2-3 \mathrm{~h}$ interval between

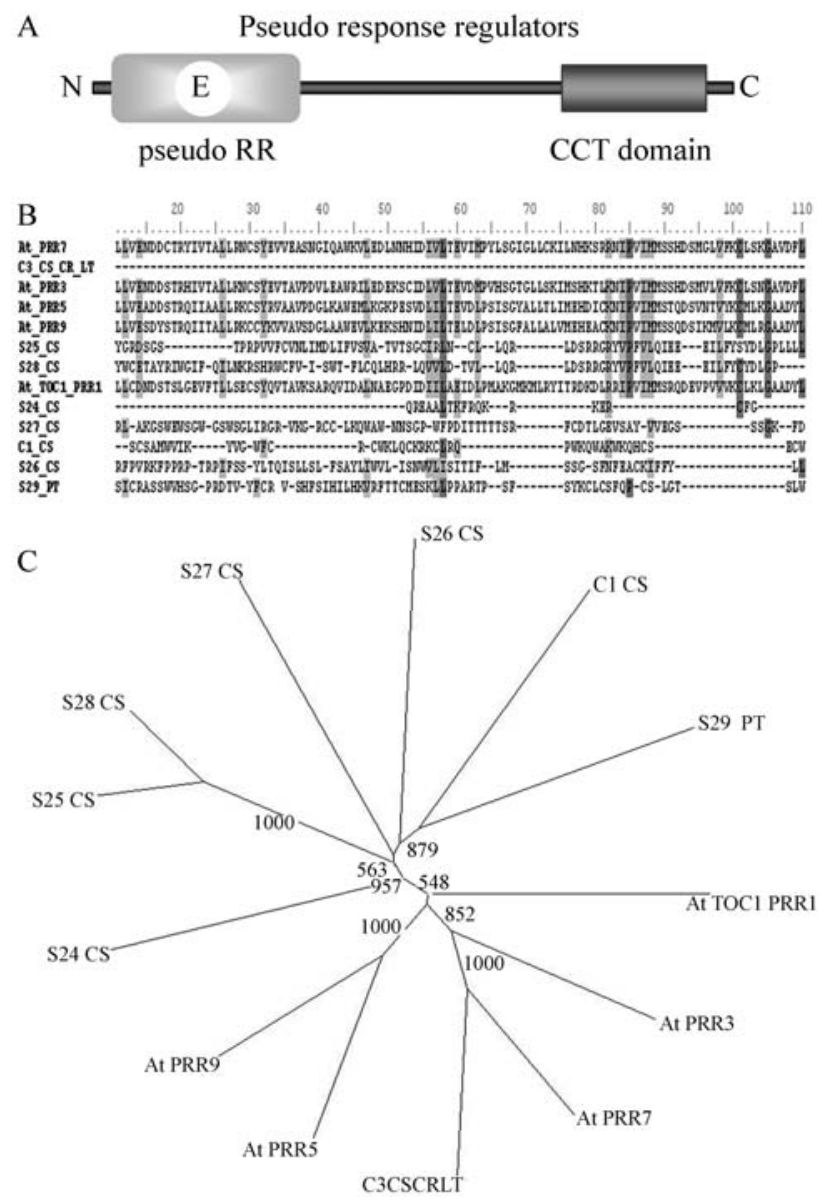

Figure 4 - Pseudo response regulator family of circadian clock components in citrus. A. Schematic representation of the domain structure of PRR proteins lacking the invariant phospho-accepting asparagine (replaced by a glutamate residue). B. Amino acid alignment of the pseudo-response regulator domain of circadian clock PRR proteins. $\mathbf{C}$. Phylogenetic analysis of citrus and $A$. thaliana PRR family. Neighbor-joining unrooted tree for citrus deduced amino acid and Arabidopsis full length sequences were aligned with ClustalX. Bootstrap values are indicated inside each branch. At, Arabidopsis thaliana; C Number, contig number; S Number, singlet number; CS, Citrus sinensis; CR, Citrus reticulata; LT, Citrus latifolia, PT, Poncirus trifoliata. 
them (Murakami et al., 2005). Such sequential induction is preceded by $C C A 1$ transcriptional activation, resulting in waves of sequential expression of CCA1-PRR9/7/5/3/1 (Mizuno and Nakamichi, 2005). These phenomena were referred to as "circadian waves of the PRR quintet"; however, the biological function of the coordinated induction of $C C A 1$ and $P R R$ transcription remains elusive. The presence of the "circadian waves of the PRR quintet" in citrus remains to be investigated. TOC1/PRR1 and PRR9 have been demonstrated to participate in phytochromemediated photomorphogenesis regulation (Makino et al., 2001; Ito et al., 2003; Más et al., 2003a; Ito et al., 2005). The expression of citrus PRR in developing fruits suggests that they might be involved in light-regulated secondary metabolism, although further functional analysis is required.

\section{Post-translation modification inducers}

The post-translational modifications involved in the circadian mechanism in Arabidopsis are protein phosphorylation, proteolytic degradation and acetylation (McClung, 2006). Four CK2-homologous transcripts have been identified in citrus transcriptome (Table 3). As observed for other oscillator components investigated, CK2 homologs are frequent in libraries obtained from fruit developmental stages and present a unique expression pattern in comparison to other putative pacemaker components; they are highly induced in developing fruits in the first and second developmental stages (Figure 3). The ZTL/FKF1/ LKP2 family, responsible for TOC1 ubiquitin-mediated degradation and regulation of photoperiodic responses, is also represented in citrus, as discussed in the accompanying paper on photoperception. Two singlets highly similar to the $\mathrm{N}$-acetylglucosamine transferase SPY protein were identified in citrus (Table 3). Thus, it is likely that the post-translational mechanisms involved in circadian clock regulation in Arabidopsis are functional in citrus.

\section{Clock outputs and integrative pathways}

The endogenous time-keeping mechanisms allow organisms to adjust the timing of crucial developmental transitions to occur at the most favorable time of the year, leading to seasonal adaptation. The mechanism of day length measuring in higher plants is based on the integration of circadian clock and light perception, at the level of $\mathrm{CO}$ protein expression and activity (Suárez-López et al., 2001; Yanovsky and Kay, 2002; Imaizumi et al., 2003; Valverde et al., 2004; Imaizumi et al., 2005).

Arabidopsis CO and its rice homolog HD1 directly regulate the expression of $F T$ and $H D 3 a$, respectively (Putterill et al., 1995; Kobayashi et al., 1999; Samach et al., 2000; Onouchi et al., 2000; Izawa et al., 2002; Kojima et al., 2002), which promote the expression of meristemidentity genes triggering the transition from vegetative to reproductive development at the shoot apical meristem
(Komeda, 2004). Fifteen $\mathrm{CO} / \mathrm{COL}$ homologs were identified in citrus transcriptome analysis (Table 3). The characteristic bipartite B-box Zn finger DNA binding domain is present at the $\mathrm{N}$ terminus of all citrus $\mathrm{CO}$ homologs (Figure 5A), although sequence divergence is observed in IIIa and IIIb sub-groups (Figure 5). A single EST contig from citrus lacked the B1 sub-domain and was clustered in the sub-group where the second B-box is replaced by a more divergent zinc-finger domain (Griffiths et al., 2003) (Figure 5B). Similarly, the signature CCT domain is highly conserved in groups I, II and IIIa and more divergent in group IIIb (Figure 5B).

Flowering requires the integration of temporal and spatial information to restrict its initiation to specific locations. Recently, Abe et al. (2005) and Wigge et al. (2005) have demonstrated that the interaction between FT expressed in the leaves and the transcription factor FLOWERING LOCUS $\underline{D}$ (FD) occurs exclusively in the shoot apex and triggers the expression of floral identity genes in the new primordial. Moreover, Huang et al. (2005) have shown that upon CO-mediated photoperiodic activation, FT mRNA is transported from the leaves to the shoot meristem via the plant vascular tissue. Therefore, $\mathrm{CO}$ action in the phloem activates $F T$ expression in leaves in a cell-autonomous manner, according to the observation that $\mathrm{CO}$ activates $F T$ expression and promotes flowering only when expressed under the control of phloem-specific promoters in the leaf, but not under control of apex-specific promoters in the shoot (An et al., 2004; Ayre and Turgeon, 2004; Valverde et al., 2004). These results indicate that the activity of $\mathrm{CO}$ is crucial for the generation of the mobile signal that originates in the leaf and is perceived in the apex at onset of flowering. Thus, leaf-produced FT activates the transcription of floral identity genes, such as APETALA1 $(A P 1)$, at the shoot apex through its interaction with a new bZIP transcription factor, FD that is expressed preferentially at the shoot apex in the region where new primordia are being generated (Abe et al., 2005; Wigge et al., 2005). Multiple lines of evidence from these studies suggest a model by which FD provides the spatial framework or timely activation of flowering by FT (Blázquez, 2005). FT and FD have been shown to physically interact in vitro and in vivo and are hypothesized to act together to activate downstream targets (Abe et al., 2005). Ectopic expression of FD caused up-regulation of $A P 1$ expression in leaves only under conditions that increase $F T$ expression, such as transfer of plants from short to long days (Wigge et al., 2005). The presence of transcripts sharing extensive sequence homology to Arabidopsis flowering integrators suggest that a similar mechanism may operate in citrus as demonstrated for other woody species (Böhlenius et al., 2006). 

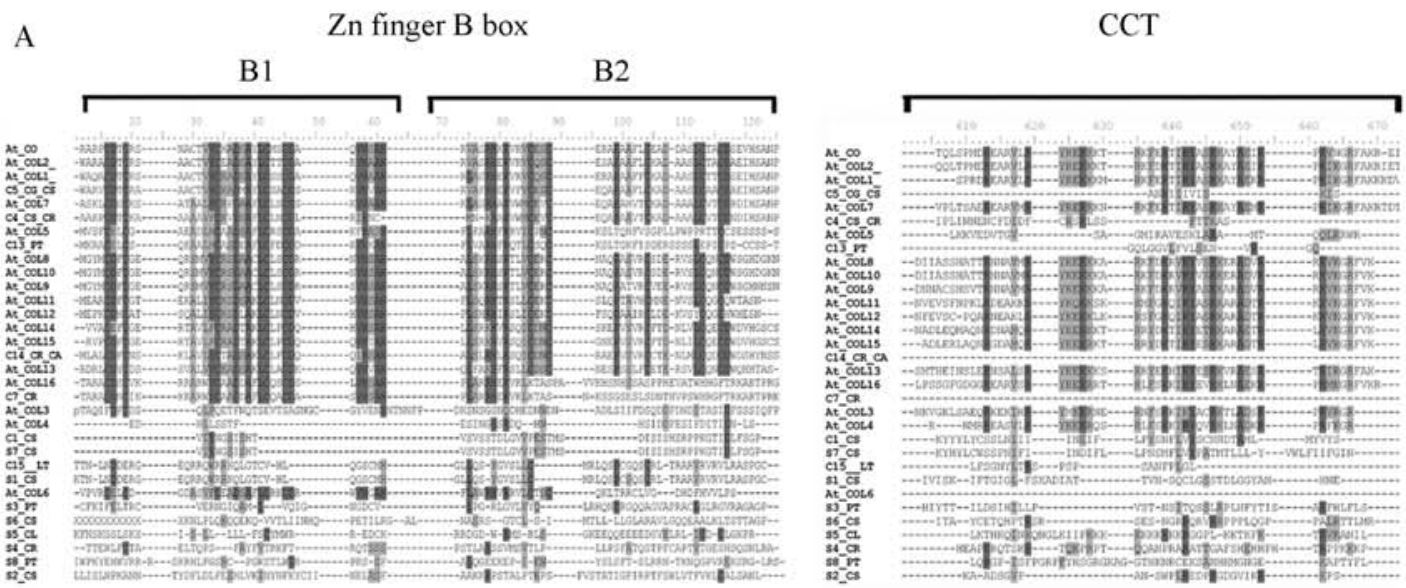

B
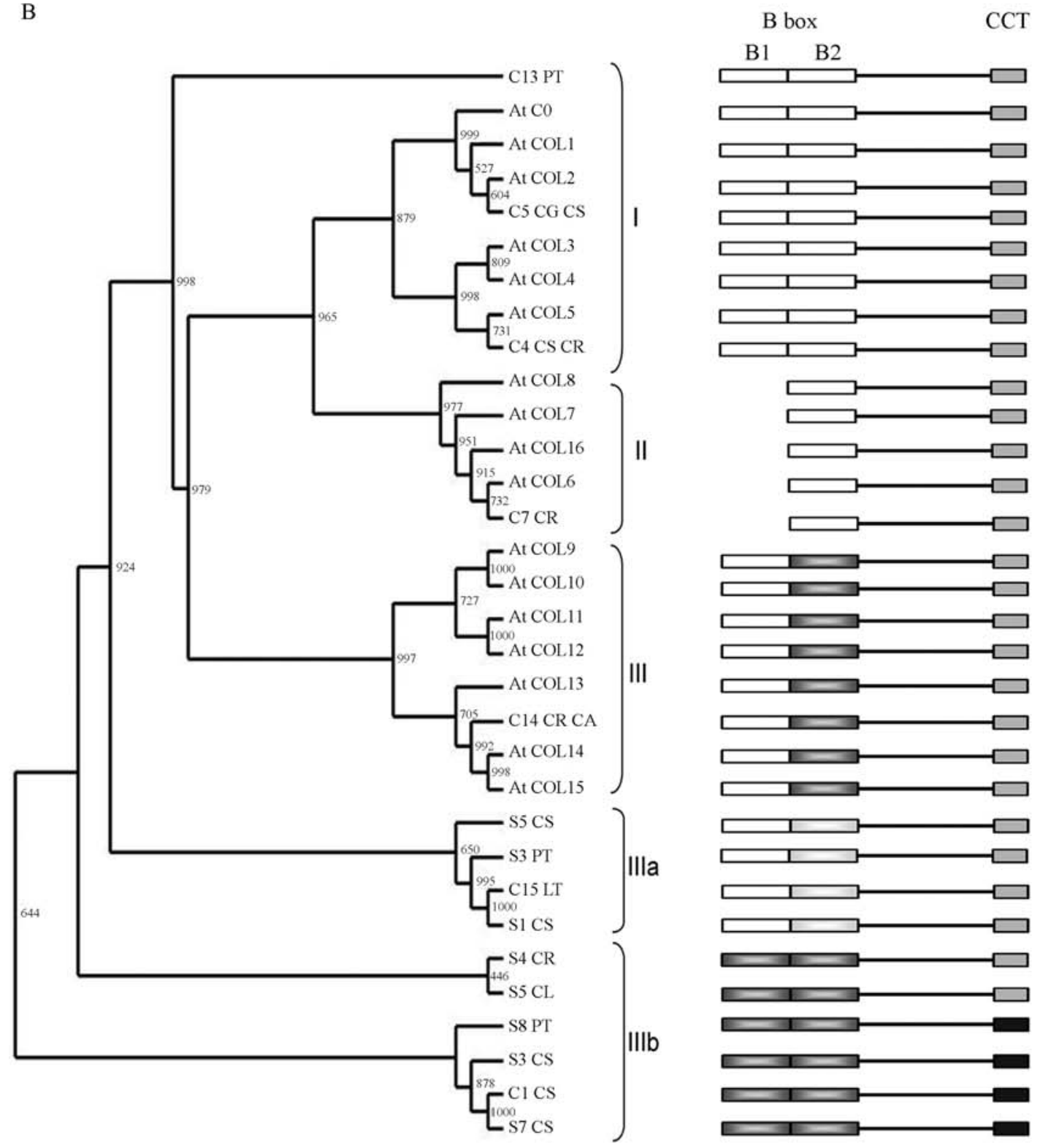

Figure 5 - CONSTANS family in citrus. A. Alignment of predicted B-box and CCT peptide domains of CONSTANS and related genes from Arabidopsis and citrus. The shaded residues represent conserved $\mathrm{C}$ residues and consensus spacing defining B-box domains. 


\section{Concluding Remarks}

A model for the endogenous oscillatory mechanism in citrus, based on comparative transcriptome and sequence conservation analysis, is proposed (Figure 6). This preliminary survey of citrus circadian clock-associated genes has provided basic information for in-depth studies relating to the endogenous time-keeping mechanism in this important plant group. Further studies of the genes identified here will also give a new perspective on the circadian regulation of economically important metabolic processes, such as secondary metabolism control. Moreover, functional characterization of the identified pathways will help to uncover the molecular basis for seasonal responses in tropical tree species. The results highlight the potential and the power of comparative genomics and in silico transcriptional profiling in perennial species.

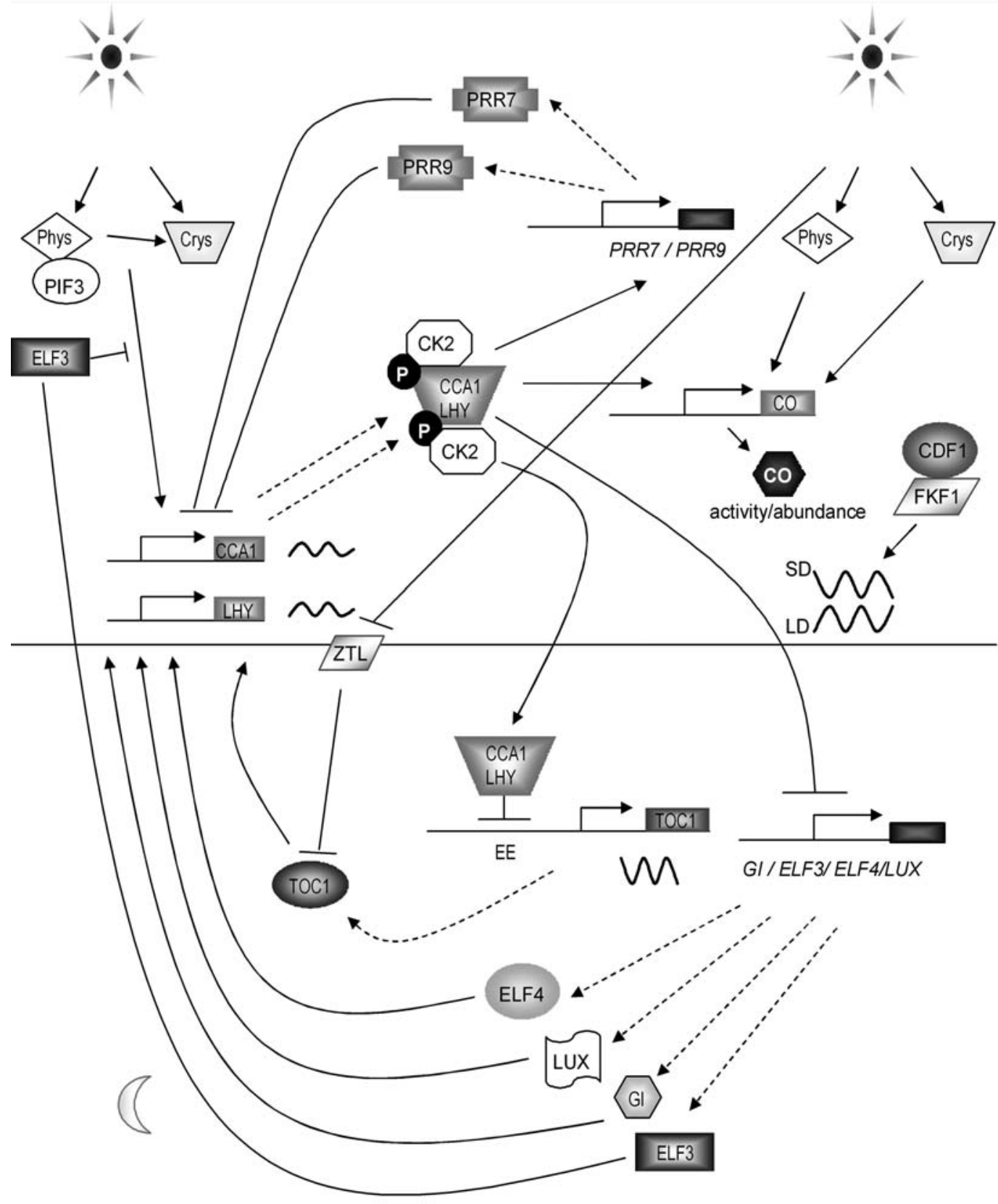

Figure 6 - Schematic overview of the endogenous time-keeping mechanism in citrus based on comparative genomic analysis. Solid boxes with italics lettering represent genes. Proteins are represented by capital letters inside oval or oblong shapes. Transcription and translation proteins are indicated by dashed lines. Protein activity is indicated by solid lines with arrowheads for positive action and perpendicular lines for negative action. The core CCA1/LHY/TOC1 feedback loop is represented by the innermost cycle whereas the ancillary loops are represented as outer loops. Phosphorylation of LHY and CCA1 by CK2 is indicated with solid circled Ps. The lower half of the figure represents activities peaking during the subjective night (moon schematic representation), whereas the superior part indicates activities peaking during the subjective day (sun schematic representation). 


\section{References}

Abe M, Kobayashi Y, Yamamoto S, Daimon Y, Yamaguchi A, Ikeda $\mathrm{Y}$, Ichinoki H, Notaguchi M, Goto $\mathrm{K}$ and Araki T (2005) FD, a bZIP protein mediating signals from the floral pathway integrator FT at the shoot apex. Science 309:1052-1056.

Abied MA and Holland D (1994) Two newly isolated genes from citrus exhibit a different pattern of diurnal expression and light response. Plant Mol Biol 26:165-173.

Alabadí D, Oyama T, Yanovsky MJ, Harmon FG, Más P and Kay SA (2001) Reciprocal regulation between TOC1 and LHY/CCA1 within the Arabidopsis circadian clock. Science 293:880-883.

Altschul SF, Madden TL, Schäffer AA, Zhang J, Zhang Z, Miller W and Lipman DJ (1997) Gapped BLAST and PSI-BLAST: A new generation of protein database search programs. Nucleic Acids Res 25:3389-3402.

An H, Roussot C, Suarez-Lopez P, Corbesier L, Vincent C, Pineiro M, Hepworth S, Mouradov A, Justin S, Turnbull CGN, et al. (2004) CONSTANS acts in the phloem to regulate a systemic signal that induces photoperiodic flowering of Arabidopsis. Development 131:3615-3626.

Ayre BG and Turgeon R (2004) Graft transmission of a floral stimulant derived from CONSTANS. Plant Physiol 135:2271-2278.

Bell-Pedersen D, Cassone VM, Earnest DJ, Golden SS, Hardin PE, Thomas TL and Zoran MJ (2005) Circadian rhythms from multiple oscillators: Lessons from diverse organisms. Nature Rev Genetics 6:4-13.

Blázquez MA (2005) Plant science. The right time and place for making flowers. Science 309:1024-1025.

Böhlenius H, Huang T, Charbonnel-Campaa L, Brunner AM, Jansson S, Strauss SH and Nilsson O (2006) CO/FT regulatory module controls timing of flowering and seasonal growth cessation in trees. Science 312:1040-1043.

Borchert R, Renner SS, Calle Z, Navarrete D, Tye A, Gautier L, Spichiger R and von Hildebrand P (2005) Photoperiodic induction of synchronous flowering near the Equator. Nature 433:627-629.

Boylan M, Douglas N and Quail PH (1994) Dominant negative suppression of Arabidopsis photoresponses by mutant phytochrome A sequences identifies spatially discrete regulatory domains in the photoreceptor. Plant Cell 6:449-460.

Carré IA (2002) ELF3: A circadian safeguard to buffer effects of light. Trends Plant Sci 7:4-6.

Cashmore AR, Jarillo JA, Wu YJ and Liu D (1999) Cryptochromes: Blue light receptors for plants and animals. Science 284:760-765.

Cerdán PD and Chory J (2003) Regulation of flowering time by light quality. Nature 423:881-885.

Chory J and Peto CA (1990) Mutations in the DET1 gene affect cell-type-specific expression of light-regulated genes and chloroplast development in Arabidopsis. Proc Natl Acad Sci USA 87:8776-8780.

Covington MF, Panda S, Liu XL, Strayer CA, Wagner DR and Kay SA (2001) ELF3 modulates resetting of the circadian clock in Arabidopsis. Plant Cell 13:1305-1315.

Daniel X, Sugano S and Tobin EM (2004) CK2 phosphorylation of CCA1 is necessary for its circadian oscillator function in Arabidopsis. Proc Natl Acad Sci USA 101:3292-3297.
Devlin PF and Kay SA (2000) Cryptochromes are required for phytochrome signaling to the circadian clock but not for rhythmicity. Plant Cell 12:2499-2510.

Doyle MR, Davis SJ, Bastow RM, McWatters HG, Kozma-Bognar L, Nagy F, Millar AJ and Amasino RM (2002) The ELF4 gene controls circadian rhythms and flowering time in Arabidopsis thaliana. Nature 419:74-77.

Edwards KD, Anderson PE, Hall A, Salathia NS, Locke JC, Lynn JR, Straume M, Smith JQ and Millar AJ (2006) FLOWERING LOCUS C mediates natural variation in the high-temperature response of the Arabidopsis circadian clock. Plant Cell 18:639-650.

Edwards KD, Lynn JR, Gyula P, Nagy F and Millar AJ (2005) Natural allelic variation in the temperature-compensation mechanisms of the Arabidopsis thaliana circadian clock. Genetics 170:387-400.

Eisen MB, Spellman PT, Brown PO and Botstein D (1998) Cluster analysis and display of genome-wide expression patterns. Proc Natl Acad Sci USA 95:14863-14868.

Endo T, Shimada T, Fujii H, Kobayashi Y, Araki T and Omura M (2005) Ectopic expression of an FT homolog from citrus confers an early flowering phenotype on trifoliate orange (Poncirus trifoliata L. Raf.). Transgenic Res 14:703-712.

Farré EM, Harmer SL, Harmon FG, Yanovsky MJ and Kay SA (2005) Overlapping and distinct roles of PRR7 and PRR9 in the Arabidopsis circadian clock. Curr Biol 15:47-54.

Fowler S, Lee K, Onouchi H, Samach A, Richardson K, Morris B, Coupland G and Putterill J (1999) GIGANTEA: A circadian clock-controlled gene that regulates photoperiodic flowering in Arabidopsis and encodes a protein with several possible membrane-spanning domains. EMBO J 18:4679-4688.

Frizzo CD, Lorenzo D and Dellacassa E (2004) Composition and seasonal variation of the essential oils from two Mandarin cultivars of southern Brazil. J Agric Food Chem 52:30363041.

Green RM and Tobin EM (1999) Loss of the circadian clockassociated protein 1 in Arabidopsis results in altered clockregulated gene expression. Proc Natl Acad Sci USA 96:4176-4179.

Griffiths S, Dunford RP, Coupland G and Laurie DA (2003) The evolution of CONSTANS-like gene families in barley, rice, and Arabidopsis. Plant Physiol 131:1855-1867.

Hall A, Bastow RM, Davis SJ, Hanano S, McWatters HG, Hibberd V, Doyle MR, Sung S, Halliday KJ and Amasino RM (2003) The TIME FOR COFFEE gene maintains the amplitude and timing of Arabidopsis circadian clocks. Plant Cell 15:2719-2729.

Han L, Mason M, Risseeuw EP, Crosby WL and Somers DE (2004) Formation of an $\mathrm{SCF}^{\mathrm{ZTL}}$ complex is required for proper regulation of circadian timing. Plant J 40:291-301.

Harmer SL and Kay SA (2005) Positive and negative factors confer phase-specific circadian regulation of transcription in Arabidopsis. Plant Cell 17:1926-1940.

Hazen SP, Schultz TF, Pruneda-Paz JL, Borevitz JO, Ecker JR and Kay SA (2005) LUX ARRHYTHMO encodes a Myb domain protein essential for circadian rhythms. Proc Natl Acad Sci USA 102:10387-10392.

He Y, Doyle MR and Amasino RM (2004) PAF1-complexmediated histone methylation of FLOWERING LOCUS C chromatin is required for the vernalization-responsive, winter-annual habit in Arabidopsis. Genes Dev 18:2774-2784. 
Hecht V, Foucher F, Ferrandiz C, Macknight R, Navarro C, Morin J, Vardy ME, Ellis N, Beltran JP, Rameau C, et al. (2005) Conservation of Arabidopsis flowering genes in model legumes. Plant Physiol 137:1420-1434.

Hicks KA, Albertson TM and Wagner DR (2001) EARLY FLOWERING 3 encodes a novel protein that regulates circadian clock function and flowering in Arabidopsis. Plant Cell 13:1281-1292.

Hicks KA, Millar AJ, Carré IA, Somers DE, Straume MD, Meeks-Wagner R and Kay SA (1996) Conditional circadian dysfunction of the Arabidopsis early-flowering 3 mutant. Science 274:790-792.

Huang T, Bohlenius H, Eriksson S, Parcy F and Nilsson O (2005) The mRNA of the Arabidopsis gene FT moves from leaf to shoot apex and induces flowering. Science 309:1694-1696.

Imaizumi T, Schultz TF, Harmon FG, Ho LA and Kay SA (2005) FKF1 F-box protein mediates cyclic degradation of a repressor of CONSTANS in Arabidopsis. Science 309:293-297.

Imaizumi T, Tran HG, Swartz TE, Briggs WR and Kay SA (2003) FKF1 is essential for photoperiodic-specific light signalling in Arabidopsis. Nature 426:302-306.

Ito S, Matsushika A, Yamada H, Sato S, Kato T, Tabata S, Yamashino T and Mizuno T (2003) Characterization of the APRR9 pseudo-response regulator belonging to the APRR1/TOC1 quintet in Arabidopsis thaliana. Plant Cell Physiol 44:1237-1245.

Ito S, Nakamichi N, Matsushika A, Fujimori T, Yamashino T and Mizuno T (2005) Molecular dissection of the promoter of the light-induced and circadian-controlled APRR9 gene encoding a clock-associated component of Arabidopsis thaliana. Biosci Biotechnol Biochem 69:382-390.

Izawa $\mathrm{T}$, Oikawa $\mathrm{T}$, Sugiyama $\mathrm{N}$, Tanisaka $\mathrm{T}$, Yano $\mathrm{M}$ and Shimamoto K (2002) Phytochrome mediates the external light signal to repress FT orthologs in photoperiodic flowering of rice. Genes Dev 16:2006-2020.

Kikis EA, Khanna R and Quail PH (2005) ELF4 is a phytochrome-regulated component of a negative-feedback loop involving the central oscillator components CCA1 and LHY. Plant J 44:300-313.

Kim W-Y, Geng R and Somers DE (2003) Circadian phasespecific degradation of the F-box protein ZTL is mediated by the proteasome. Proc Natl Acad Sci USA 100:49334938.

Kobayashi Y, Kaya H, Goto K, Iwabuchi M and Araki T (1999) A pair of related genes with antagonistic roles in mediating flowering signals. Science 286:1960-1962.

Kojima S, Takahashi Y, Kobayashi Y, Monna L, Sasaki T, Araki $\mathrm{T}$ and Yano M (2002) Hd3a, a rice ortholog of the Arabidopsis FT gene, promotes transition to flowering downstream of Hd1 under short-day conditions. Plant Cell Physiol 43:1096-1105.

Komeda Y (2004) Genetic regulation of time to flower in Arabidopsis thaliana. Annu Rev Plant Biol 55:521-535.

Lee HS and Castle WS (2001) Seasonal changes of carotenoid pigments and color in Hamlin, Earlygold, and Budd Blood orange juices. J Agric Food Chem 49:877-882.

Lin J, Rouseff RL, Barros S and Naim M (2002) Aroma composition changes in early season grapefruit juice produced from thermal concentration. J Agric Food Chem 50:813-819.

Liu XL, Covington MF, Fankhauser C, Chory J and Wagner DR (2001) ELF3 encodes a circadian clock-regulated nuclear protein that functions in an Arabidopsis PHYB signal transduction pathway. Plant Cell 13:1293-1304.

Locke JCW, Southern MM, Kozma-Bognar L, Hibberd V, Brown PE, Turner MS and Millar AJ (2005) Extension of a genetic network model by iterative experimentation and mathematical analysis. Mol Syst Biol 1, doi/10.1038/msb4100018, http://www.nature.com/msb/journal/v1/n1/full/ msb4100018.html.

Makino S, Kiba T, Imamura A, Hanaki N, Nakamura A, Suzuki T, Taniguchi M, Ueguchi C, Sugiyama T and Mizuno T (2000) Genes encoding pseudo-response regulators: Insight into His-to-Asp phosphorelay and circadian rhythm in Arabidopsis thaliana. Plant Cell Physiol 41:791-803.

Makino S, Matsushika A, Kojima M, Oda Y and Mizuno T (2001) Light response of the circadian waves of the APRR1/TOC1 quintet: When does the quintet start singing rhythmically in Arabidopsis? Plant Cell Physiol 42:334-339.

Más P, Alabadí D, Yanovsky MJ, Oyama T and Kay SA (2003a) Dual role of TOC1 in the control of circadian and photomorphogenic responses in Arabidopsis. Plant Cell 15:223-236.

Más P, Devlin PF, Panda S and Kay SA (2000) Functional interaction of PHYTOCHROME B and CRYPTOCHROME 2. Nature 408:207-211.

Más P, Kim W-Y, Somers DE and Kay SA (2003b) Targeted degradation of TOC1 by ZTL modulates circadian function in Arabidopsis thaliana. Nature 426:567-570.

Matsushika A, Makino S, Kojima M and Mizuno T (2000) Circadian waves of expression of the APRR1/TOC1 family of pseudo-response regulators in Arabidopsis thaliana: Insight into the plant circadian clock. Plant Cell Physiol 41:10021012.

Matsushika A, Makino S, Kojima M, Yamashino T and Mizuno T (2002) The APRR1/TOC1 quintet implicated in circadian rhythms of Arabidopsis thaliana: II. Characterization with CCA1-overexpressing plants. Plant Cell Physiol 43:118122.

McClung CR (2006) Plant circadian rhythms. Plant Cell 18:792803.

McWatters HG, Bastow RM, Hall A and Millar AJ (2000) The ELF3 Zeitnehmer regulates light signaling to the circadian clock. Nature 408:716-20.

Michael TP and McClung CR (2002) Phase-specific circadian clock regulatory elements in Arabidopsis thaliana. Plant Physiol 130:627-638.

Millar AJ (2004) Input signals to the plant circadian clock. J Exp Bot 55:277-283.

Mizoguchi T, Wheatley K, Hanzawa Y, Wright L, Mizoguchi M, Song HR, Carré IA and Coupland G (2002) LHY and CCA1 are partially redundant genes required to maintain circadian rhythms in Arabidopsis. Dev Cell 2:629-641.

Mizuno T and Nakamichi N (2005) Pseudo-response regulators (PRRs) or true oscillator components (TOCs). Plant Cell Physiol 46:677-685.

Murakami M, Matsushika A, Ashikari M, Yamashino T and Mizuno T (2005) Circadian-associated rice pseudo response regulators (OsPRRs): Insight into the control of flowering time. Biosci Biotechnol Biochem 69:410-414.

Murakami M, Yamashino T and Mizuno T (2004) Characterization of circadian-associated APRR3 Pseudo-Response Regulator belonging to the APRR1/TOC1 quintet in Arabidopsis thaliana. Plant Cell Physiol 45:645-650. 
Nakamichi N, Kita M, Ito S, Sato E, Yamashino T and Mizuno T (2005a) PSEUDO-RESPONSE REGULATORS, PRR9, PRR7 and PRR5, together play essential roles close to the circadian clock of Arabidopsis thaliana. Plant Cell Physiol 46:686-698.

Nakamichi N, Kita M, Ito S, Sato E, Yamashino T and Mizuno T (2005b) The Arabidopsis pseudo-response regulators, PRR5 and PRR7, coordinately play essential roles for circadian clock function. Plant Cell Physiol 46:609-619.

Nelson DC, Lasswell J, Rogg LE, Cohen MA and Bartel B (2000) $F K F 1$, a clock-controlled gene that regulates the transition to flowering in Arabidopsis. Cell 101:331-340.

Ni M, Tepperman JM and Quail PH (1999) Binding of phytochrome B to its nuclear signalling partner PIF3 is reversibly induced by light. Nature 400:781-784.

Onai K and Ishiura M (2005) PHYTOCLOCK 1 encoding a novel GARP protein essential for the Arabidopsis circadian clock. Genes Cells 10:963-972.

Onouchi H, Igeno MI, Perilleux C, Graves K and Coupland G (2000) Mutagenesis of plants overexpressing CONSTANS demonstrates novel interactions among Arabidopsis flowering-time genes. Plant Cell 12:885-900.

Park DH, Somers DE, Kim YS, Choy YH, Li HK, Soh MS, Kim HJ, Kay SA and Nam HG (1999) Control of circadian rhythms and photoperiodic flowering by the Arabidopsis GIGANTEA gene. Science 285:1579-1582.

Pena L, Martin-Trillo M, Juarez J, Pina JA, Navarro L and Martinez-Zapater JM (2001) Constitutive expression of Arabidopsis LEAFY or APETALA1 genes in citrus reduces their generation time. Nat Biotechnol 19:263-267.

Pillitteri LJ, Lovatt CJ and Walling LL (2004) Isolation and characterization of a TERMINAL FLOWER homolog and its correlation with juvenility in citrus. Plant Physiol 135:15401551 .

Putterill J, Robson F, Lee K, Simon R and Coupland G (1995) The CONSTANS gene of Arabidopsis promotes flowering and encodes a protein showing similarities to zinc finger transcription factors. Cell 80:847-857.

Robson F, Costa MM, Hepworth SR, Vizir I, Pineiro M, Reeves PH, Putterill J and Coupland G (2001) Functional importance of conserved domains in the flowering-time gene CONSTANS demonstrated by analysis of mutant alleles and transgenic plants. Plant J 28:619-631.

Salomé PA and McClung CR (2005) PSEUDO-RESPONSE REGULATOR 7 and 9 are partially redundant genes essential for the temperature responsiveness of the Arabidopsis circadian clock. Plant Cell 17:791-803.

Salomé PA, Michael TP, Kearns EV, Fett-Neto AG, Sharrock RA and McClung CR (2002) The out of phase 1 mutant defines a role for PHYB in circadian phase control in Arabidopsis. Plant Physiol 129:1674-1685.

Samach A, Onouchi H, Gold SE, Ditta GS, Schwarz-Sommer Z, Yanofsky MF and Coupland G (2000) Distinct roles of CONSTANS target genes in reproductive development of Arabidopsis. Science 288:1613-1616.

Santelli RV and Siviero F (2001) A search for homologues of plant photoreceptor genes and their signaling partners in the sugarcane expressed sequence tag (Sucest) database. Genet Mol Biol 24:49-53.

Schaffer R, Ramsay N, Samach A, Corden S, Putterill J, Carré IA and Coupland G (1998) The late elongated hypocotyl muta- tion of Arabidopsis disrupts circadian rhythms and the photoperiodic control of flowering. Cell 93:1219-1229.

Schultz TF, Kiyosue T, Yanovsky M, Wada M and Kay SA (2001) A role for LKP2 in the circadian clock of Arabidopsis. Plant Cell 13:2659-2670.

Sharrock RA and Clack T (2002) Patterns of expression and normalized levels of the five Arabidopsis phytochromes. Plant Physiol 130:442-456.

Sharrock RA and Quail PH (1989) Novel phytochrome sequences in Arabidopsis thaliana: Structure, evolution, and differential expression of a plant regulatory photoreceptor family. Genes Dev 3:1745-1757.

Smith NG and Wareing PF (1972) The rooting of actively growing and dormant leafy cuttings in relation to endogenous hormone levels and photoperiod. New Phytol 71:483-500.

Somers DE, Devlin PF and Kay SA (1998) Phytochromes and cryptochromes in the entrainment of the Arabidopsis circadian clock. Science 282:1488-1490.

Somers DE, Schultz TF, Milnamow M and Kay SA (2000) ZEITLUPE encodes a novel clock-associated PAS protein from Arabidopsis. Cell 101:319-329.

Song H-R and Carré IA (2005) DET1 regulates the proteasomal degradation of LHY, a component of the Arabidopsis circadian clock. Plant Mol Biol 57:761-771.

Staiger D, Allenbach L, Salathia N, Fiechter V, Davis SJ, Millar AJ, Chory J and Fankhauser C (2003). The Arabidopsis $S R R 1$ gene mediates phyB signaling and is required for normal circadian clock function. Genes Dev 17:256-268.

Strayer CA, Oyama T, Schultz TF, Raman R, Somers DE, Más P, Panda S, Kreps JA and Kay SA (2000) Cloning of the Arabidopsis clock gene TOC1, an autoregulatory response regulator homolog. Science 289:768-771.

Suárez-López P, Wheatley K, Robson F, Onouchi H, Valverde F and Coupland G (2001) CONSTANS mediates between the circadian clock and the control of flowering in Arabidopsis. Nature 410:1116-1120.

Sugano S, Andronis C, Green RM, Wang Z-Y and Tobin EM (1998) Protein kinase CK2 interacts with and phosphorylates the Arabidopsis circadian clock-associated 1 protein. Proc Natl Acad Sci USA 95:11020-11025.

Sugano S, Andronis C, Ong MS, Green RM and Tobin EM (1999) The protein kinase CK2 is involved in regulation of circadian rhythms in Arabidopsis. Proc Natl Acad Sci USA 96:12362-12366

Thompson JD, Gibson TJ, Plewniak F, Jeanmougin F and Higgins DG (1997) The ClustalX windows interface: Flexible strategies for multiple sequence alignment aided by quality analysis tools. Nucleic Acids Res 24:4876-4882.

Tóth R, Kevei E, Hall A, Millar AJ, Nagy F and Kozma-Bognar L (2001) Circadian clock-regulated expression of phytochrome and cryptochrome genes in Arabidopsis. Plant Physiol 127:1607-1616.

Tseng T-S, Salomé PA, McClung CR and Olszewski NE (2004) SPINDLY and GIGANTEA interact and act in Arabidopsis thaliana pathways involved in light responses, flowering and rhythms in leaf movements. Plant Cell 16:1550-1563.

Valverde F, Mouradov A, Soppe W, Ravenscroft D, Samach A and Coupland G (2004) Photoreceptor regulation of CONSTANS protein in photoperiodic flowering. Science 303:1003-1006. 
Wang ZY and Tobin EM (1998) Constitutive expression of the circadian clock associated 1 (CCA1) gene disrupts circadian rhythms and suppresses its own expression. Cell 93:12071217.

Wigge PA, Kim MC, Jaeger KE, Busch W, Schmid M, Lohmann JU and Weigel D (2005) Integration of spatial and temporal information during floral induction in Arabidopsis. Science 309:1056-1059.

Yano M, Kojima S, Takahashi Y, Lin H and Sasaki T (2001) Genetic control of flowering time in rice, a short-day plant. Plant Physiol 127:1425-1429.

Yanovsky MJ and Kay SA (2002) Molecular basis of seasonal time measurement in Arabidopsis. Nature 419:308-312.

Yanovsky MJ, Mazzella MA and Casal JJ (2000) A quadruple photoreceptor mutant still keeps track of time. Curr Biol 10:1013-1015.

\section{Internet Resources}

Citrus Biotechnology Laboratory, http://citest.centrodecitricul tura.br (September 13, 2006).

Cluster v.2.11 Software, http://rana.lbl.gov/EisenSoftware.htm.

DNASTAR Lasergene Software, http://www.dnastar.com/web/ index.php.

European Bioinformatics Institute-European Molecular Biology Laboratory (EMBL-EBI), www.ebi.ac.uk/interpro/ (September 04, 2006).

Expert Protein Analysis System (ExPaSy), http://www.expasy. org/prosite/ and http://www.us.expasy.org/sprot/ (October 05, 2006).
Gene Ontology (GO), http://www.godatabase.org/cgi-bin/amigo/ go.cgi (October 23, 2006).

PAUP* 4.0b10 Software, http://paup.csit.fsu.edu/.

Protein Families (Pfam), http://www.sanger.ac.uk/Software/ Pfam/ (October 15, 2006).

PSIGNFIT Software, http://www.bootstrap-software.org/.

The Institute for Genomic Research (TIGR) Arabidopsis thaliana v.13.0 Gene Ontology Assignments, http://compbio.dfci. harvard.edu/tgi/cgi-bin/tgi/GO_browser.pl?species = Arabi dopsis\&gi_dir $=$ agi $($ October 23,2006$)$.

Tree View v.1.6 Software, http://rana.lbl.gov/EisenSoftware.htm.

\section{Supplementary Material}

Table 1S- Citrus reads constituting contigs and singlets with homology to genes involved in the input pathway to the circadian clock in Arabidopsis thaliana.

Table $2 \mathrm{~S}$ - Citrus reads constituting contigs and singlets with homology to genes involved in the central oscillator of the circadian clock in Arabidopsis thaliana.

Table 3S - Citrus reads constituting contigs and singlets with homology to genes involved in the integration of the circadian clock mechanism and the generation of output responses in Arabidopsis thaliana.

This material is available as part of the online article from http://www.scielo.br/gmb.

Associate Editor: Alessandra Alves de Souza 
Table 1S - Citrus reads constituting contigs and singlets with homology to genes involved in the input pathway to the circadian clock in Arabidopsis thaliana.

\begin{tabular}{|c|c|c|}
\hline Gene & Contig / Singlet & $\operatorname{Read}(\mathrm{s})$ \\
\hline \multirow[t]{2}{*}{$C R Y 1$} & $\mathrm{C} 10-\mathrm{CR}$ & CR05-C3-702-066-D11-CT, CR05-C3-702-061-F10-CT \\
\hline & S1-CS & CS00-C1-100-038-A12-CT \\
\hline \multirow[t]{2}{*}{ PHYA } & S2-CS & CS00-C3-701-101-C11-CT \\
\hline & S3 - CS & CS00-C3-705-056-G06-CT \\
\hline \multirow[t]{2}{*}{$P H Y B$} & S4-CR & CR05-C1-102-036-H07-CT \\
\hline & S5-CS & CS12-G8-000-003-D03-CT \\
\hline \multirow[t]{3}{*}{ FKF1 } & $\mathrm{C} 5-\mathrm{CS}$ & CS00-C1-102-025-G12-CT, CS00-C1-102-019-A06-CT \\
\hline & C6-CR/CS & CR05-C3-700-019-F11-EU, CS00-C1-102-053-E02-CT, \\
\hline & & CS00-C2-003-018-C11-CT \\
\hline$L K P 2$ & S6-CS & CS00-C3-702-030-D01-CT \\
\hline$Z T L$ & C5-CS & CS00-C1-102-025-G12-CT, CS00-C1-102-019-A06-CT \\
\hline \multirow[t]{4}{*}{ ELF3 } & $\mathrm{C} 1-\mathrm{CS} / \mathrm{CR}$ & CS00-C3-702-072-C10-CT, CR05-C1-100-007-E05-CT \\
\hline & C2- CR & CR05-C3-701-025-F10-CT, CR05-C1-102-098-H10-CT \\
\hline & S7-CA & CA26-C1-002-082-B12-CT \\
\hline & S8-PT & PT11-C1-900-009-D09-CT \\
\hline \multirow[t]{8}{*}{$G I$} & $\mathrm{C} 1-\mathrm{CS}$ & CSO0-C3-703-068-A06-CT, CS00-C1-102-030-G09-CT, CSO0- \\
\hline & & C3-702-018-C07-CT, CS00-C1-102-024-G11-CT, CS00-C3- \\
\hline & & 705-013-A05-CT, CS00-C3-705-019-F09-CT \\
\hline & $\mathrm{C} 2-\mathrm{CS} / \mathrm{CR}$ & CS00-C3-703-056-C05-CT, CR05-C1-100-083-B11-CT \\
\hline & $\mathrm{C} 3-\mathrm{CG} / \mathrm{CR} / \mathrm{CS}$ & CG32-C1-003-008-F11-CT, CG32-C1-003-008-F10-CT, \\
\hline & & CR05-C3-701-28-12-CT, CS00-C2-003-033-G06-CT \\
\hline & S9-CS & CS00-C1-102-069-D09-CT \\
\hline & S10-CR & CR05-C3-702-072-F10-CT \\
\hline PIF3 & C6-CG/LT & CG32-C1-003-054-B09-CT, LT33-C1-003-071-A08-CT \\
\hline
\end{tabular}


Table 2S - Citrus reads constituting contigs and singlets with homology to genes involved in the central oscillator of the circadian clock in Arabidopsis thaliana.

\begin{tabular}{|c|c|c|}
\hline Gene & Contig / Singlet & $\operatorname{Read}(s)$ \\
\hline \multirow[t]{10}{*}{$\overline{C C A 1}$} & $\mathrm{C} 3-\mathrm{CS}$ & CS00-C3-700-046-B06-CT, CS00-C3-702-034-H03-CT \\
\hline & $\mathrm{C} 7-\mathrm{CS} / \mathrm{PT}$ & CS00-C3-702-101-A04-CT, CS12-C1-001-007-F05-CT, \\
\hline & & CS12-C1-001-012-C11-CT, PT11-C1-900-055-F12-CT, CS00- \\
\hline & & C1-100-122-C11-CT, CS00-C1-100-103-A02-UV, PT11-C1- \\
\hline & & 901-048-D05-CT, PT11-C1-900-043-B06-CT \\
\hline & C16-PT & PT11-C1-900-047-B09-CT, PT11-C1-901-054-F01-CT, PT11- \\
\hline & & C1-900-080-D04-CT., PT11-C1-900-048-E05-CT \\
\hline & C18-CS/CR & CS00-C1-650-031-A10-CT, CS00-C3-701-030-A11-CT, \\
\hline & & CR05-C1-100-043-F03-CT, CR05-C1-102-049-H08-CT \\
\hline & S11-CR & CR05-C1-103-085-D10-CT \\
\hline \multirow[t]{6}{*}{$C K 2$} & $\mathrm{C} 2-\mathrm{CR} / \mathrm{CS}$ & CR05-C3-701-006-F08-CT, CS00-C1-100-096-D01-UV, \\
\hline & & CS00-C3-700-002-H11-CT, CS00-C3-700-006-G03-CT, \\
\hline & & CS00-C3-700-008-H05-CT, CS00-C3-700-074-F07-CT \\
\hline & $\mathrm{S} 12-\mathrm{CG}$ & CG32-C1-003-100-G07-CT \\
\hline & S13-CR & CR05-C1-103-023-A01-CT \\
\hline & S14-CS & CS00-C3-700-023-H06-CT \\
\hline \multirow[t]{2}{*}{ DET1/FUS2 } & S15-CS & CS00-C3-700-016-F11-CT \\
\hline & S16-LT & LT33-C1-003-085-H03-CT \\
\hline \multirow[t]{3}{*}{ ELF4 } & S17-CR & CR05-C1-102-100-G07-CT \\
\hline & S18-CS & CS13-C1-001-024-G11-CT \\
\hline & S19-PT & PT11-C2-300-083-B03-CT \\
\hline$L H Y$ & C11-CS/CR & CS00-C1-650-047-H07-CT, CS00-C1-100-085-E03-EU, \\
\hline
\end{tabular}


CR05-C3-701-026-B05-CT, CS00-C3-705-032-D04-CT, CS00-C1-102-032-E11-CT, CR05-C3-701-082-D12-CT, CS00-C1-100-042-E09-CT, CS00-C1-100-042-E10-CT, CS00-C3-700-001-D05-CT, CS00-C1-100-081-H04-CT, CS00-C3-702-046-G10-CT, CS00-C1-100-016-G11-CT, CS00-C1-100-115-F08-CT, CS00-C1-100-041-D04-CT, CS00-C3-704-082-A10-CT, CS00-C3-705-032-E11-CT, CS00-C3-702-074-D03-CT, CR05-C3-701-001-G09-CT, CR05-C3-701-030-A10-CT, CS00-C3-702-052-G12-CT, CR05-C1-100-076-B12-CT, CS00-C1-101-009-H10-CT C14-PT PT11-C1-901-005-C05-CT, PT11-C1-901-061-A10-CT S20-PT PT11-C1-900-008-A04-CT S21-CR CR05-C1-102-100-G07-CT

$L U X \quad$ C1-CS CS00-C3-701-070-D03-CT, CS00-C3-702-058-D08-CT, IPCLI CS00-C3-700-045-C11-CT, CS00-C3-705-096-B11-CT, family CS00-C3-705-096-H07-CT, CS00-C3-701-031-F06-CT C2- CS/PT CS00-C1-100-065-D11-CT, PT11-C1-901-020-E04-CT, CS00-C1-100-105-A06-EU S22-CR CR05-C3-700-068-H07-CT S23-CR CR05-C3-701-021-A12-CT

PRR1/ S24-CS CS00-C1-100-073-A05-CT TOC1

PRR3 C3-CS/CR/LT CR05-C3-701-012-G04-CT, CS00-C3-703-079-A01-CT, CS00-C3-703-079-F01-CT, LT33-C1-003-018-F05-CT.F, CS00-C3-705-007-F07-CT, CR05-C3-701-082-C01-CT, 
CS00-C3-705-002-H03-CT, CS00-C3-702-052-A12-CT

S25-CS

CS00-C1-102-110-C02-CT

S26-CS

CS00-C3-701-087-D07-UV

PRR7

S27-CS

CS00-C3-702-097-G04-CT

S28-CS

CS00-C3-703-091-H07-CT

S29-PT

PT11-C1-901-059-F10-CT

PRR9

C1-CS

CS00-C3-702-035-D05-CT, CS00-C3-700-053-E01-CT

$\mathrm{C} 2-\mathrm{CS} / \mathrm{CR}$

CS00-C3-704-006-B05-CT, CS00-C3-704-009-E12-CT,

CR05-C1-102-033-A01-CT

$S P Y$

$\mathrm{C} 1-\mathrm{CS} / \mathrm{CR}$

CR05-C3-702-099-H11-CT, CS00-C2-003-046-H03-CT, CR05-C1-100-064-E08-CT, CR05-C3-700-108-H02-CT,

CR05-C1-100-007-B05-CT, CS00-C3-705-041-D11-CT , CS00-C3-702-023-B02-CT

S30-CR

CR05-C1-103-015-E09-CT

S31-CS

CS00-C1-102-059-G10-CT 
Table 3S - Citrus reads constituting contigs and singlets with homology to genes involved in the integration of the circadian clock mechanism and the generation of output responses Arabidopsis thaliana.

\begin{tabular}{|c|c|c|}
\hline Gene & Contig / Singlet & $\operatorname{Read}(\mathrm{s})$ \\
\hline$C D F 1$ and & C4-CS/PT & CS00-C1-100-122-G01-CT, PT11-C1-900-024-E10-CT, \\
\hline$C D F$ & & CS00-C1-100-014-F05-CT \\
\hline \multirow[t]{5}{*}{ family } & $\mathrm{C} 10-\mathrm{CA} / \mathrm{CR}$ & CA26-C1-002-099-H09-CT, CR05-C1-102-048-C08-CT \\
\hline & S1-CS & CS00-C1-100-077-E09-CT \\
\hline & S2-PT & PT11-C1-900-023-D03-CT \\
\hline & S3-PT & PT11-C1-900-057-F03-CT \\
\hline & S4-PT & PT11-C1-901-002-A06-CT \\
\hline \multirow[t]{2}{*}{$\mathrm{CO}$} & $\mathrm{C} 5-\mathrm{CG} / \mathrm{CS}$ & CS00-C1-100-086-A06-CT, CS00-C1-100-008-H03-CT, \\
\hline & & CS12-C1-001-026-C04-CT, CS12-C1-001-027-D01-CT \\
\hline COL1 and & $\mathrm{C} 4-\mathrm{CS} / \mathrm{CR}$ & CS00-C1-101-087-B11-UV, CS00-C1-101-065-A09-CT, CS00- \\
\hline$C O L$ & & C1-100-123-E03-CT, CS00-C1-100-045-C06-CT, CS00-C1- \\
\hline \multirow[t]{9}{*}{ family } & & 100-011-G06-CT, CS00-C3-700-072-G04-CT, CS00-C1-101- \\
\hline & & 102-D07-EU, CS00-C1-101-031-H07-CT, \\
\hline & & CS00-C1-100-041-B09-CT, CS00-C1-101-006-F05-UV, CSO0- \\
\hline & & C1-650-027-B06-CT, CS00-C1-650-027-E11-CT, CS00-C1- \\
\hline & & 100-061-E06-UV, CR05-C3-701-035-A11-CT, CS00-C3-702- \\
\hline & & 013-B06-CT, CS00-C1-650-002-C12-CT, CSO0-C1-650-021- \\
\hline & & F04-CT, CS00-C1-100-099-A01-UV, CR05-C3-701-056-E06- \\
\hline & & CT, CR05-C1-103-084-D07-CT, CS00-C3-705-019-C05-CT, \\
\hline & & CS00-C3-705-022-G05-CT, CS00-C1-102-088-F03-CT, CR05- \\
\hline
\end{tabular}




\begin{tabular}{|c|c|c|}
\hline & $\mathrm{C} 1-\mathrm{CS}$ & CS13-C1-001-040-D02-CT, CS00-C1-100-008-C08-CT \\
\hline & C7-CR & CR05-C3-701-028-A11-CT, CR05-C1-100-028-C11-CT \\
\hline & C13-PT & PT11-C9-005-035-E08-CT, PT11-C1-901-063-G03-CT \\
\hline & $\mathrm{C} 14-\mathrm{CR} / \mathrm{CA}$ & CR05-C3-701-040-A06-CT, CR05-C3-700-021-A02-UV, \\
\hline & & CA26-C1-002-073-H02-CT \\
\hline & C15-LT & LT33-C1-003-105-G01-CT, LT33-C1-003-106-C12-CT \\
\hline & S1-CS & CS00-C1-101-022-A10-CT \\
\hline & S2-CS & CS00-C1-101-004-F12-CT \\
\hline & S3-PT & PT11-C1-900-069-G04-CT \\
\hline & S4-CR & CR05-C1-102-087-D12-CT \\
\hline & S5-CL & CL06-C4-500-018-F06-CT \\
\hline & S6-CS & CS00-C1-102-043-C11-CT \\
\hline & S7-CS & CS13-C1-001-040-D02-CT \\
\hline & S8-PT & PT11-C1-901-084-D12-CT \\
\hline ELF7 & $\mathrm{C} 1-\mathrm{CR} / \mathrm{LT}$ & CR05-C3-701-022-H04-CT, CR05-C1-103-040-G06-CT, LT33- \\
\hline & & C1-003-056-B09-CT \\
\hline$E L F 8$ & $\mathrm{C} 1-\mathrm{CS}$ & CS00-C1-101-036-F06-CT, CS00-C1-101-013-H07-CT, \\
\hline & & CS00-C1-102-019-G06-CT, CS00-C3-700-074-H01-CT \\
\hline$F D$ & S1-CG & CG32-C1-003-009-B06-CT \\
\hline$F L C$ & $\mathrm{C} 1-\mathrm{CS}$ & CS12-C1-001-012-H03-CT, CS00-C1-102-067-G11-CT, CS00- \\
\hline & & C1-102-066-G08-CT, CS00-C1-102-065-H11-CT, CS00-C1- \\
\hline & & 101-023-C09-CT \\
\hline & S1-CS & CS00-C1-101-021-F12-CT \\
\hline & S2-CS & CS12-C1-001-009-G11-CT \\
\hline FT/ & S1-CS & CS00-C3-704-020-B11-CT \\
\hline
\end{tabular}


TSF

PFT1 C2-CS/CR CS00-C1-101-049-D12-CT, CS00-C3-701-019-D08-CT, CS00-

C1-650-028-B01-CT, CS12-G8-000-105-E11-CT, CR05-C1-

103-095-D04-CT, CR05-C1-103-074-G03-CT

SOC1 S1-PT PT11-C1-900-082-A11-CT

S2-PT PT11-C1-900-063-G11-CT 